\title{
Co-aggregation and secondary nucleation in the life cycle of human prolactin/galanin functional amyloids
}

D.Chatterjee $^{1}$, R.S. Jacob ${ }^{1}$, S. Ray ${ }^{1}$, A. Navalkar ${ }^{1}$, N. Singh ${ }^{1}$, S. Sengupta ${ }^{1}$, L. Gadhe ${ }^{1}$, P.

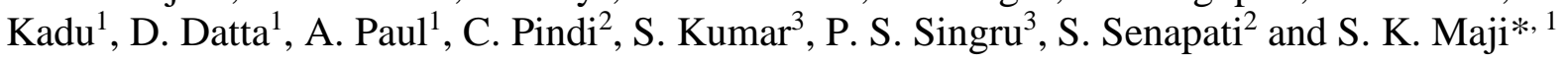

${ }^{1}$ Department of Biosciences and Bioengineering, Indian Institute of Technology Bombay, Mumbai, India

${ }^{2}$ Department of Biotechnology, Indian Institute of Technology Madras, Chennai 600036, India

${ }^{3}$ School of Biological Sciences, National Institute of Science Education and Research, Bhubaneswar, India

*Correspondence: Prof. Samir K. Maji

Department of Biosciences and Bioengineering, IIT Bombay, Powai, Mumbai-400076, India.

Telephone: +91-22-2576-7774.Fax:+91-22-2572 3480. E-mail: samirmaji@iitb.ac.in 


\begin{abstract}
Synergistic-aggregation and cross-seeding by two different amyloid proteins/peptides are well evident in various neurological disorders. However, this phenomenon is not well studied in functional amyloid aggregation. Here, we show Prolactin (PRL) is associated with lactation in mammals and neuropeptide galanin (GAL), which are co-stored in the lactotrophs facilitates the synergic aggregation in the absence of secretory granules helper molecules glycosaminoglycans (GAGS). Interestingly, although each partner possesses homotypic seeding ability, a unidirectional cross-seeding of GAL aggregation can be mediated by PRL seeds. The specificity of co-aggregation by PRL and GAL along with unidirectional crossseeding suggests tight regulation of functional amyloid formation during co-storage of these hormones in secretory granule biogenesis of female rat lactotrophs. Further mixed fibrils release the constituent functional hormone much faster than the corresponding individual amyloid formed in presence of GAGs, suggesting that co-aggregation of functionally distant hormones might have evolved for efficient storage, synergistic and rapid release of both hormones upon stimulation. The co-aggregation and cross seeding by two different hormones of completely different structures and sequences (PRL and GAL) suggest a novel mechanism of heterologous amyloid formation both in disease and functional amyloids.
\end{abstract}




\section{Introduction}

Protein/peptide misfolding, aggregation, and amyloid formation is associated with various neurological disorders such as Alzheimer's and Parkinson's ${ }^{1,2}$. However, several studies have suggested that amyloid formation is also associated with the native biological function of the host organism. The protein fibrils formed to aid in the functionality of the host organisms are called functional amyloids ${ }^{3-7}$. For example, Pmel-17 amyloid fibrils template melanin polymerization inside melanosomes ${ }^{8-10}$; Curli fibrils of $E$. coli support the organism to adhere to a surface and also help their colonization process inside biofilms ${ }^{11,12}$. In this line, another very interesting aspect is the formation of functional amyloid by protein/peptide hormones (such as prolactin (PRL) and galanin (GAL)) during their storage inside the secretory granules (SGs) of pituitary ${ }^{13,14}$. The amyloid formation, in this case, not only enriches the protein concentration to serve as a protease-resistant protein/peptide storage depot but is also able to release the functional monomeric proteins upon dilution and $\mathrm{pH}$ changes ${ }^{13-17}$.

Protein/peptide aggregation and amyloid formation generally follow nucleation-dependent polymerization mechanism ${ }^{18-20}$, where protein/peptide slowly associates to form aggregation competent nuclei (in the lag phase of aggregation) $)^{21-23}$. Once formed, the aggregation competent nuclei further recruit the monomeric counterpart for their growth into mature amyloid fibrils (elongation phase) ${ }^{21-23}$. The progression of aggregation eventually reaches a steady-state equilibrium between the fibrils and monomeric protein (stationary phase) ${ }^{19,24}$. Recent evidences however suggest that fragmentation/elongation and secondary nucleation may contribute to a significant decrease in the lag-time of aggregation, similar to an external addition of preformed nuclei in amyloid growth reaction (seeding) ${ }^{25-27}$. Although homotypic aggregation and seeding is the most favored mechanism of protein aggregation and amyloid formation, synergistic aggregation (co-aggregation) by two different proteins/peptides and heterologous seeding are also suggested to be involved in many neurodegenerative disorders $^{28-32}$. This is one of the possible mechanisms by which one disease aggravates the other disease such as Alzheimer's disease and Type 2 diabetes and Alzheimer's disease and Parkinson's disease ${ }^{33,34}$.

PRL and GAL secretion is synergistic and promoted by common secretagogues ${ }^{35-37}$. PRL/GAL co-storage has been also reported in the anterior pituitary of female rats or estrogen-treated male rats $^{38}$. Here, we investigate the synergistic aggregation and amyloid formation by these hormones for their secretory storage and release from secretory granules. Indeed, both of these hormones are co-stored in the female rat pituitary and possess amyloid- 
like characteristics. In this study, we show that both hormones not only engage in homotypic amyloid aggregation in the presence of respective GAGs as helper molecules but also synergistically aggregate (in absence of GAGs) to form heterotypic amyloid containing PRL and GAL as suggested by double immunoelectron microscopy. Intriguingly, cross-seeding with PRL fibril seeds resulted in fibrillation of GAL. However, GAL fibrils could not seed PRL monomers for fibrillation. This suggests a tightly controlled regulation of hormone amyloids for their homotopic and heterotypic storage. Furthermore, our in vitro release assay showed faster release of functional monomers by heterotypic, hybrid amyloid (PRL+GAL) compared to homotypic counterparts. This supports the storage and release are highly controlled and conserved in pituitary tissue for the optimum function to be served.

\section{Results}

PRL and GAL are co-stored as amyloids in SGs of the anterior pituitary of female rat.

Previously it was shown that PRL and GAL co-store in the anterior pituitary of female rats or estrogen-treated male rats ${ }^{38-40}$. PRL is a $23 \mathrm{kDa}$ protein (Supplementary Table 1) and consists of four-helix bundles comprising residues 14-42 (helix 1), 78-104 (helix 2), 110-138 (helix 3) and 160-194 (helix 4) ${ }^{41,42}$. The helix bundles are spaced with 3 loop regions (Fig 1a). On the other hand, GAL is a small unstructured neuropeptide (Supplementary Table 1), which is 30 residues in length ${ }^{43,44}$ (Fig 1a). PRL showed amyloid-prone sequences predicted by $\mathrm{TANGO}^{45}$; GAL, however, did not contain any amyloid-prone sequences (Fig 1b-c). Since previously it was established that protein/peptide hormones (including PRL and GAL) can be stored as amyloids inside the $(\mathrm{SGs})^{13}$, we tested whether PRL and GAL are colocalized at anterior pituitary in the amyloid state or not. To examine this possibility, immunofluorescence study of female rat pituitary was performed using anti-GAL (green) and anti-PRL (red) antibodies (see material and methods). Our results showed that PRL and GAL were substantially co-localized in the pituitary tissue (Fig 1d) indicating their costorage inside the SGs. Further, to test whether both PRL and GAL remain in the amyloid state, we performed immunostaining of the tissue with amyloid specific antibody OC along with Thioflavin-S (ThioS) ${ }^{46}$ (Fig 1e, Supplementary Figure 1). Double immunofluorescence of PRL and GAL along with amyloid specific OC antibody showed strong colocalization suggesting both the hormones are in the amyloid state (Fig 1e), which was also observed in ThioS staining (Supplementary Figure 1). Altogether, our data confirmed that both PRL and GAL are co-stored as amyloid aggregates in the female rat pituitary. 
bioRxiv preprint doi: https://doi.org/10.1101/2021.08.31.458467; this version posted September 1, 2021. The copyright holder for this preprint (which was not certified by peer review) is the author/funder, who has granted bioRxiv a license to display the preprint in perpetuity. It is made available under aCC-BY-NC-ND 4.0 International license.

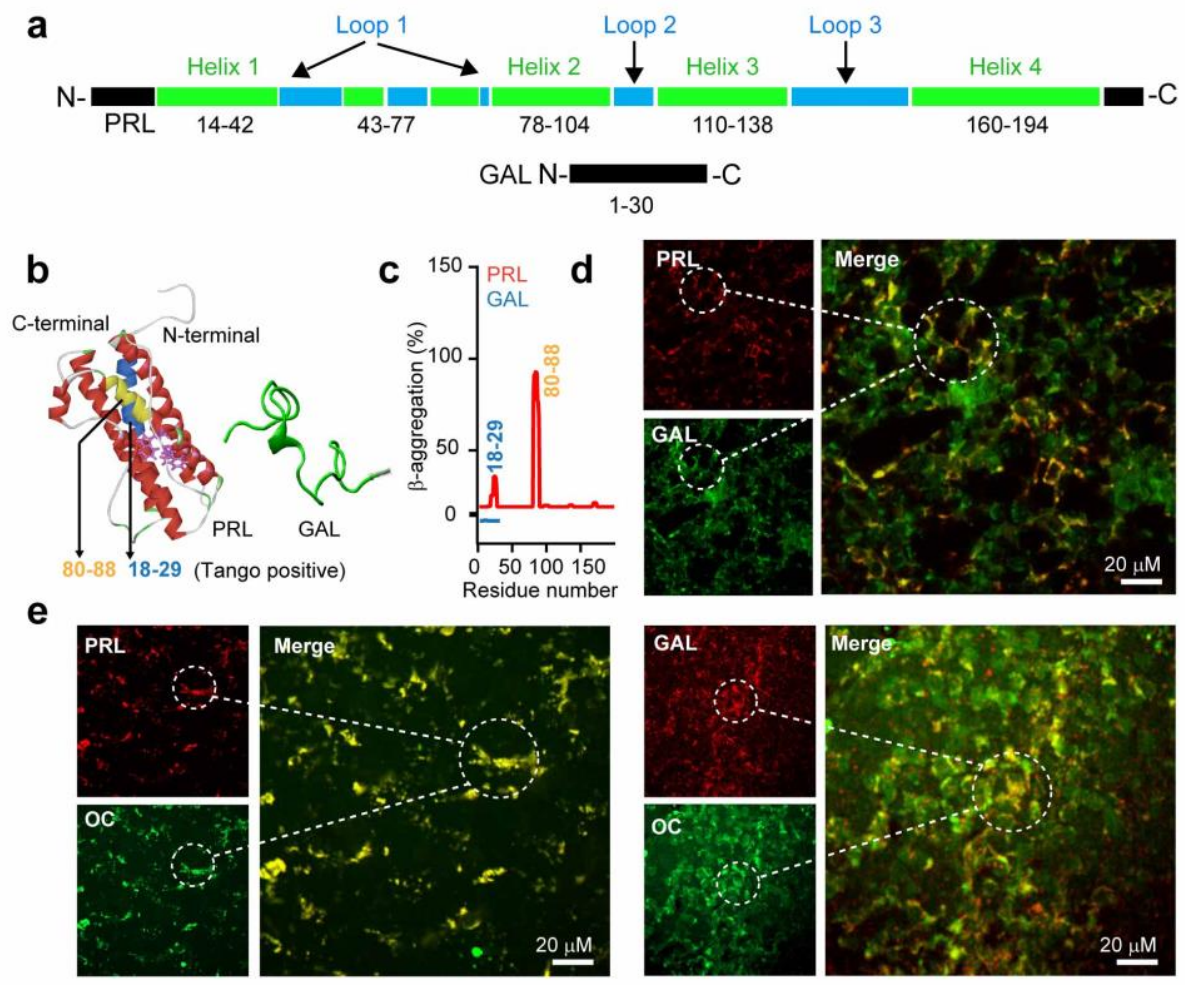

Fig 1: Amyloid propensity and co-storage of PRL and GAL.

a. Schematic showing amino acid sequence and secondary structures of PRL \& GAL with different color codes. (Upper panel): PRL is 191 amino acids in length and contains a four-helix bundle (green). The short helix and loop regions are also represented between helix-1 and helix 2 (shown in green and blue colors, respectively). (Lower panel): GAL showing 30 residue peptide with no definite secondary structure ${ }^{43,44}$. b. (Left panel) The three-dimensional structure (obtained in Pymol) ${ }^{41}$ of PRL showing its major helices and two tryptophan residues (shown in purple) (PDB ID: 1RW5). (Right panel): Natively unstructured conformation of GAL is also shown. c. TANGO algorithm showing the aggregation-prone residues of PRL and GAL at pH 6.0 (SG relevant $\mathrm{pH}$ ). The residues 18-29 and 8088 of PRL showing amyloid aggregation potential. However, TANGO analysis of GAL revealed no amyloid aggregation propensity. Immunofluorescence studies showing (d) colocalization of PRL (red) and GAL (green) in the female rat anterior pituitary. (e) left panel. Colocalization of amyloid fibrils (OC, green) and PRL (red) and amyloid fibrils (OC, green) and GAL (red) (Right panel)) in the anterior pituitary. The merged photomicrographs showing colocalization (yellow). The experiments (d-e) are performed three times with similar observations.

\section{In vitro amyloid aggregation kinetics and synergistic co-fibrils of PRL and GAL.}

The colocalization of both PRL and GAL in the amyloid state suggested that PRL and GAL might interact with each other and might co-aggregate inside the same SGs. We hypothesized that there could be 4 possibilities (case 1-4) when PRL and GAL are co-stored as amyloids (Fig 2a). The two proteins can form separate filaments and these filaments can be incorporated into heterogeneous fibrils (case 1). They can form completely separate, homogeneous fibrils either by PRL or GAL or by both hormones (case 2). It is also possible that the PRL and GAL together can form the fibril forming unit to form heterogeneous 
bioRxiv preprint doi: https://doi.org/10.1101/2021.08.31.458467; this version posted September 1, 2021. The copyright holder for this preprint (which was not certified by peer review) is the author/funder, who has granted bioRxiv a license to display the preprint in perpetuity. It is made available under aCC-BY-NC-ND 4.0 International license.

amyloid fibrils (case 3); and lastly, the PRL monomers can simply adhere to the GAL fibril (case 4) and vice-versa (case 5).

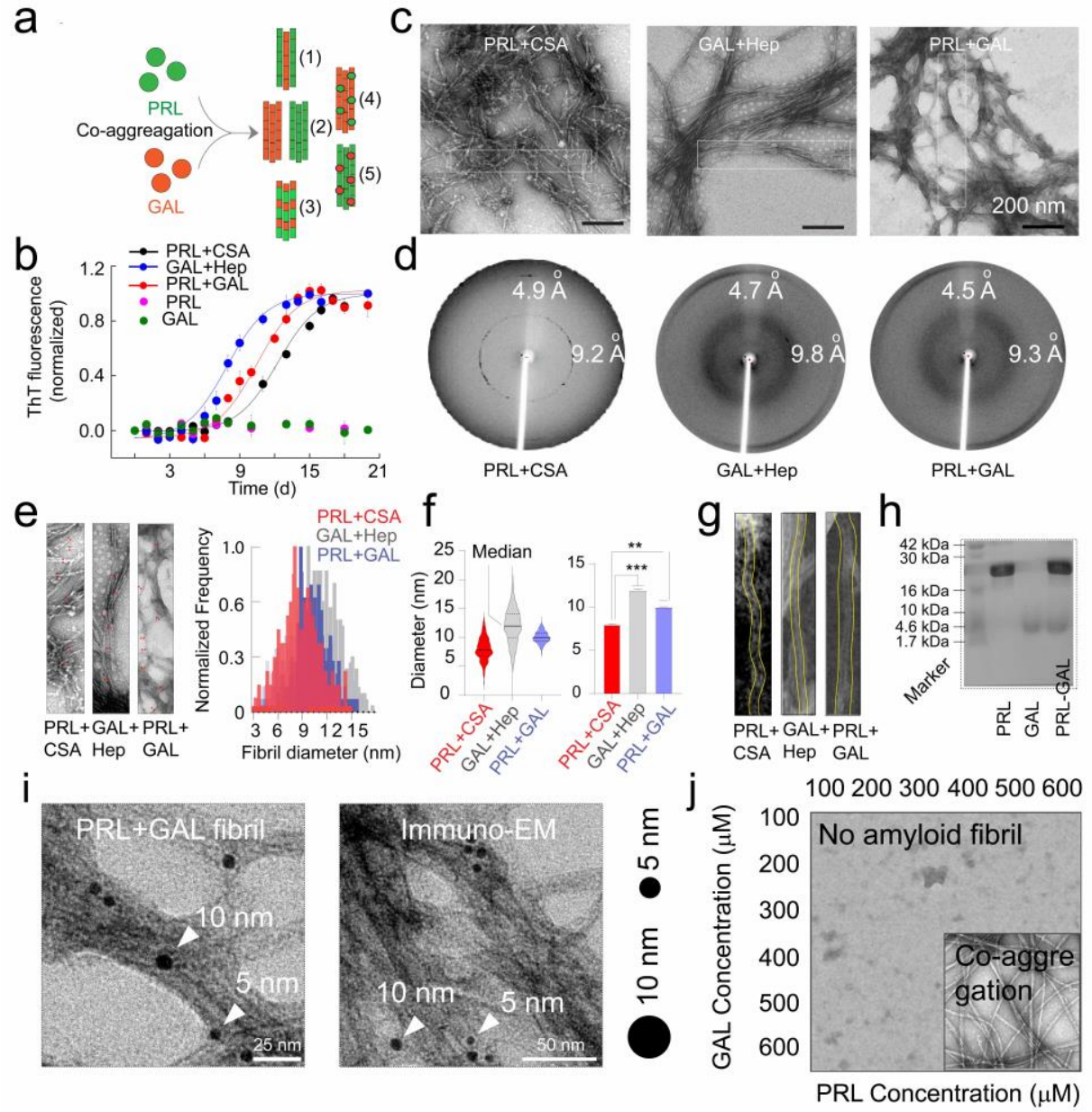

Fig 2: Amyloid aggregation kinetics \& co-fibril formation by PRL and GAL:

a. Schematic showing different possibilities for the formation of PRL-GAL co-fibril. b. Normalized ThT fluorescence intensity over time showing faster aggregation kinetics for GAL-Hep followed by PRL-GAL and PRL-CSA. The experiment is performed three times with similar results. Values represent mean \pm SEM. c. TEM images showing amyloid fibrils for PRL-CSA, GAL-Hep, and PRLGAL after 15 days of incubation. Representative images are shown. The dotted box marks are the representative area from which the fibril diameters are measured. d. XRD of PRL-CSA, GAL-Hep, and PRL-GAL fibrils at day 15 showing $\sim 4.7 \AA$ meridional and $\sim 10 \AA$ equatorial reflections, as commonly seen for most amyloid fibrils ${ }^{47,48}$. e. (Left panel) Representative TEM images showing fibril diameter measured at random positions (marked with red arrows) on individual fibrils. (Right panel) The normalized frequency distribution of fibril diameters of PRL+CSA, GAL+Hep, and PRL+GAL fibrils is shown. 200 data points are collected for individual samples for $n=3$ independent experiments. f. (Left panel) Median values of different fibril diameters are shown with violin plots. (Right panel) Average values of different fibril diameters are shown. Values represent mean \pm SD for $\mathrm{n}=3$ independent experiments. The statistical significance $(* * * \mathrm{p} \leq 0.001, * * \mathrm{p} \leq 0.01)$ is calculated by one-way ANOVA followed by an SNK post hoc test with a 95\% confidence interval. g. Representative TEM images of PRL-CSA, GAL-Hep, and PRL-GAL fibrils (scale bar- $200 \mathrm{~nm}$ ). From a single fibril, 200 data points are collected along the length to calculate the diameter. h. SDS-PAGE depicting two bands for isolated aggregates from the PRL-GAL mixture (lane 3). The two bands 
correspond to PRL and GAL, which suggests that the isolated aggregates are composed of both PRL and GAL. i. Amyloid fibrils obtained from the PRL-GAL mixture showing $10 \mathrm{~nm}$ gold particles (against GAL primary) and $5 \mathrm{~nm}$ gold particles (against PRL primary), confirming synergistic cofibril formation by PRL and GAL (Left and Right panel). The experiment is performed three times with similar observations. j. Schematic representation of incubation of PRL and GAL at various concentrations showing optimum concentration is required to initiate PRL-GAL co-aggregation.

To explore these possibilities, we asked whether two hormones with completely different sequences, length and without any sequence similarity/identity (Supplementary Figure 2) can co-assemble to form hybrid fibrils. To do this, we mixed the 1:1 molar ratio of both the hormones, incubated at $37{ }^{\circ} \mathrm{C}$, and followed the amyloid formation by Thioflavin-T (ThT) binding and CD spectroscopy for 15 days (Fig 2b, Supplementary Figure 2). As a control, both hormones were incubated alone. Since PRL and GAL are known to form amyloid in presence of chondroitin sulfate A (CSA) and Heparin (Hep) ${ }^{13}$, we also incubated both the hormones in presence of their respective GAGs as positive controls (Fig $\mathbf{2 b}$, Supplementary Figure 2). As expected, both PRL and GAL showed fibril formation in the presence of CSA and Hep. No ThT binding was observed by either PRL or GAL in absence of GAGs (Fig $\mathbf{2 b}$, Supplementary Figure 2).

Strikingly, the mixture of both the hormones showed high ThT binding during time indicating amyloid formation (Fig 2b, Supplementary Figure 2).CD spectroscopy showed a substantial decrease of helical content by PRL-GAL and PRL-CSA during aggregation. Notably, PRL is known to form amyloid without structural conversion to $\beta$-sheet ${ }^{13}$. On the other hand, GALHep showed random coil to $\beta$-sheet conversion during amyloid formation (Supplementary Figure 2). The lag times of aggregation for PRL-CSA, PRL-GAL, and GAL-Hep were calculated as $\sim 9.2$ days, $\sim 8.8$ days, and $\sim 7$ days, respectively (Supplementary Figure 2), suggesting that PRL-GAL aggregated faster than PRL-CSA. Moreover, PRL-GAL aggregates showed fibril like morphology under TEM (Fig 2c), strong apple-green/golden birefringence under cross-polarized light (Supplementary Figure 3), exhibited cross $\beta$-sheet diffraction patterns ${ }^{47,48}(\sim 4.7 \AA$ for inter-strand and $\sim 9.8 \AA$ for inter-sheet) (Fig 2d) and FTIR peaks corresponding to $\beta$-sheet structure $^{49,50}$ (Supplementary Figure 4). Similar observations supporting amyloid structure were also obtained for PRL-CSA and GAL-Hep aggregates (Fig 2c-d and Supplementary Figures 2-4).

Although there are various possibilities regarding how PRL-GAL can form fibrils (any of the component hormones can form fibrils separately or together, as described in Fig 2a), we analyzed the frequency distribution of the fibril diameters from TEM images. Fibril diameters were measured from a particular sample (n=200 randomized points) (Fig 2e, left panel) and 
the normalized frequency distribution was plotted (Fig 2e, right panel). Our data indicated that the diameter of PRL-CSA fibrils was least (Fig 2e, right panel) having a median value of $\sim 7.5 \mathrm{~nm}$ (Fig 2f, left panel) and an average of $\sim 7.8 \mathrm{~nm}$ (Fig 2f, right panel). The diameter of GAL-Hep fibrils was highest (Fig 2e, right panel) having a median value of $\sim 12.3 \mathrm{~nm}$ (Fig 2f, left panel) and an average of $\sim 12 \mathrm{~nm}$ (Fig 2f, right panel). Intriguingly, the PRL-GAL fibrils showed an intermediate diameter (median 10 nm, average 9.8 nm) (Fig 2e-f). This data suggest that PRL-GAL fibrils might be a new type of hybrid fibrils, which is neither similar to GAL nor PRL fibrils. We further analyzed the diameter from the same fibril bundle along its length. Consistent with our random point analysis (Fig 2e-f), we found that the diameter of a single PRL-CSA fibril was indeed the lowest (average $7 \mathrm{~nm}$ ) followed by PRL-GAL (average $10 \mathrm{~nm}$ ) and GAL-Hep (average 12 nm) fibril (Fig 2g, Supplementary Figure 2). Our data indicate that PRL-GAL fibrils possess unique morphology, which could be due to the incorporation of both PRL as well as GAL molecules into the same fibril (cofibril).

To further analyze whether both PRL and GAL are part of the same insoluble fibril fraction, we isolated the fibrils using centrifugation and performed SDS-PAGE. The presence of two bands corresponding to PRL and GAL indicated that both PRL and GAL formed fibrils when co-incubated together (Fig 2h). Further, we performed immuno-electron microscopy (Immuno EM) with the PRL-GAL fibrils in the presence of secondary antibodies against PRL and GAL attached with $5 \mathrm{~nm}$ and $10 \mathrm{~nm}$ gold nanoparticles, respectively (Fig 2i). Colocalization of both 5 and $10 \mathrm{~nm}$ nanoparticles within the same fibril bundle confirmed that both PRL and GAL were part of the same fibril bundle (Fig 2i). However, due to extensive bundling of fibrils, it remains to be seen whether PRL and GAL are incorporated in the same filament or not.

Since $400 \mu \mathrm{M}$ (each) PRL-GAL could undergo aggregation even in the absence of any helper molecules, we wanted to understand the optimum concentration and stoichiometry for PRLGAL co-aggregation. To do this, we chose increasing concentration of PRL and GAL in an orthogonal (X-Y axis) manner (from 100-600 $\mu$ M, each) (Fig 2j, Supplementary Fig 5). All combinations of this concentration regime of hormone mixture were incubated for 15 days. We found $\geq 1: 1$ ratio and $\geq 400 \mu \mathrm{M}$ concentration are required for mixed fibrils formation (experimentally feasible time scale) as determined by ThT binding and electron microscope study (Fig 2j, Supplementary Fig 5). The data suggest that the equimolar ratio of PRL and GAL can co-assemble to form hybrid fibrils in a concentration-dependent manner. 
bioRxiv preprint doi: https://doi.org/10.1101/2021.08.31.458467; this version posted September 1,2021 . The copyright holder for this preprint (which was not certified by peer review) is the author/funder, who has granted bioRxiv a license to display the preprint in perpetuity. It is made available under aCC-BY-NC-ND 4.0 International license.

\section{Cross-seeding of PRL and GAL}

Amyloid aggregation is a nucleation-dependent polymerization process ${ }^{18,19}$. It is well-known fact that the presence of preformed nuclei of amyloid (also called "seeds") greatly affects the kinetics of aggregation of monomers ${ }^{25,51}$. Since PRL and GAL co-aggregate into mixed amyloids, we asked whether both PRL and GAL could cross-seed ${ }^{25,51-54}$ to induce amyloid aggregation of each other and help their possible storage in SGs. To do that, we performed both homotypic (PRL monomer+PRL seed; GAL monomer+GALseed) and heterotypic seeding (PRL monomer+GAL seed and GAL monomer+PRL seed).
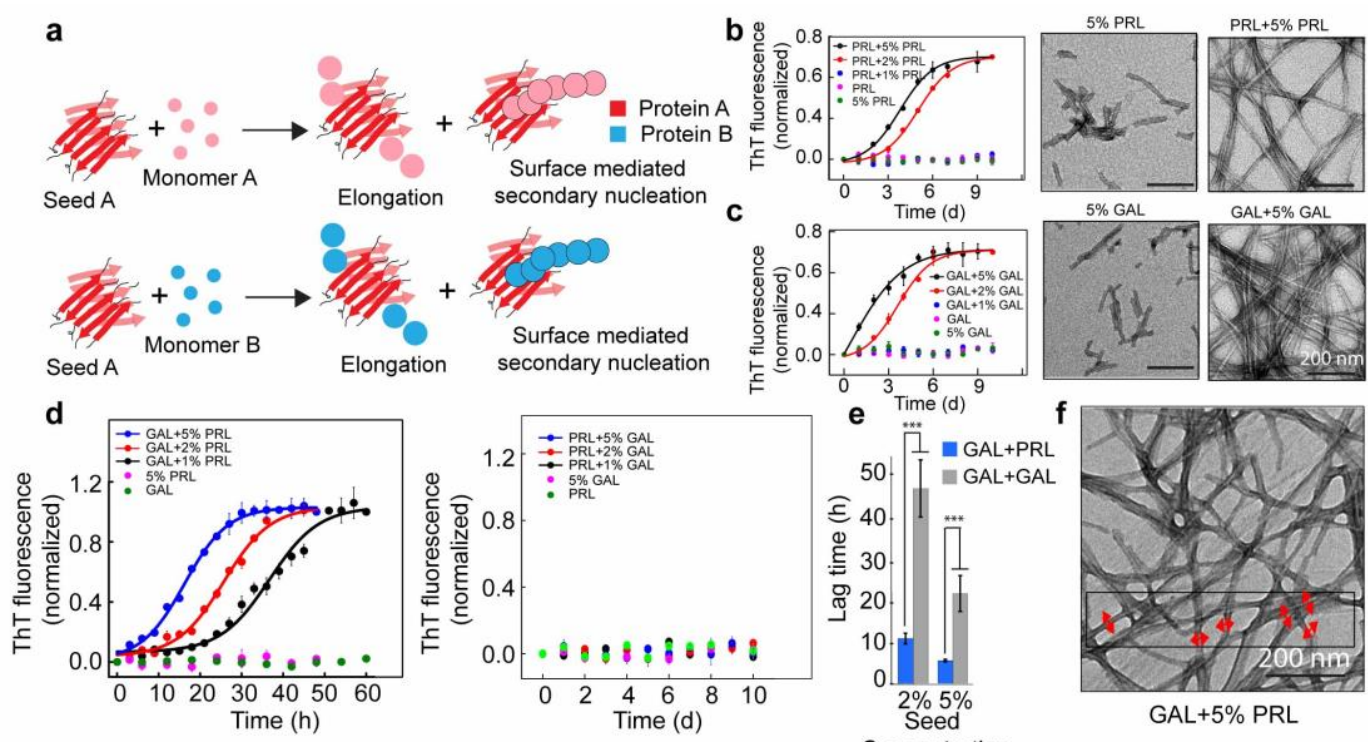

9
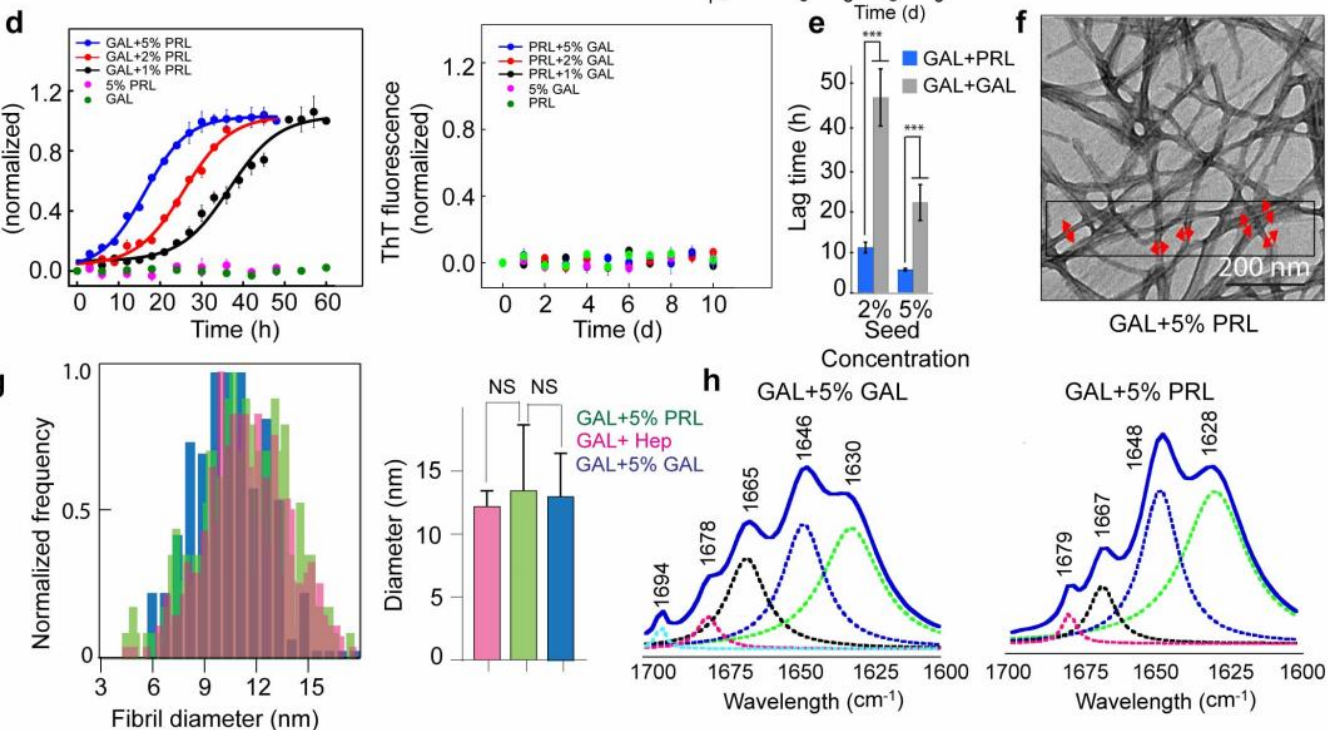

$\mathrm{h}$

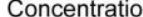

$\mathrm{GAL}+5 \% \mathrm{PRL}$

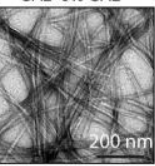

GAL $+5 \%$ GAL

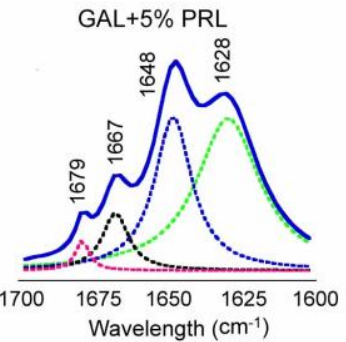

\section{Fig 3: Seeding and cross-seeding of PRL and GAL:}

a. Schematic showing possible homo and hetero seeding with fibril elongation and surface-mediated secondary nucleation mechanism for seed-mediated fibril growth. (b-c). homo-seeding of PRL and GAL. (left panel) PRL and GAL homo-seeding. Normalized ThT fluorescence intensity values with time indicating aggregation of PRL and in the presence of different concentrations of PRL seeds and GAL seeds respectively ( $2 \%$ and 5\% v/v). Only seeds and only PRL/GAL was used as controls. (right panel) The corresponding EM images of PRL/GAL seeds alone and PRL/GAL monomer in presence of 5\% PRL/GAL seeds showing fibrils formation by PRL/GAL homo-seeding. d. Crossseeding of PRL and GAL. (left panel) Normalized ThT fluorescence intensity values with time indicating aggregation of GAL in the presence of different concentrations of PRL seeds $(1 \%, 2 \%$, and $5 \% \mathrm{v} / \mathrm{v})$. However, PRL in presence of different percentages of GAL seeds does not show any aggregation (right panel). Only seed and only GAL/PRL were used as controls where no aggregation 
is observed. e. The lag times of GAL aggregation in presence of $2 \%$ and 5\% v/v PRL seeds and GAL seeds are compared. The values represent mean \pm SEM. The significance $(* * * p \leq 0.001)$ is calculated using one-way ANOVA followed by an SNK post hoc test with a 95\% confidence interval. f. TEM images of GAL fibrils formed in presence of PRL seeds are shown. GAL fibrils formed in presence of $5 \%(\mathrm{v} / \mathrm{v})$ PRL seeds are analyzed for frequency distribution (red arrows indicating the diameter of the fibrils measured for analysis). g. (left panel) Normalized frequency distribution of fibril diameter showing GAL fibrils formed in presence of PRL seeds have a similar diameter to GAL+Hep fibrils. 200 random data points from different individual fibrils were collected from $n=3$ independent experiments for the frequency distribution analysis. (right panel) Average values of different fibril diameters are shown. Values represent mean \pm SD for $n=3$ independent experiments. The statistical significance $\left(* * * \mathrm{p} \leq 0.001,{ }^{* *} \mathrm{p} \leq 0.01\right)$ is calculated by one-way ANOVA followed by an SNK post hoc test with a $95 \%$ confidence interval. h. FTIR spectra showing fibrils of GAL+5\% GAL seed and GAL $+5 \%$ PRL seed are of similar secondary structure.

Preformed fibrils were sonicated to obtain PRL and GAL amyloid fibril seeds (see materials and methods). 1\%, 2\%, and 5\%(v/v) of PRL and GAL seeds were mixed with freshly prepared $400 \mu \mathrm{M}$ PRL and GAL, respectively, and incubated with slight agitation at $37^{\circ} \mathrm{C}$ for homotypic seeding (Fig 3b, Supplementary Figure 6). Our ThT fluorescence data showed accelerated aggregation by both PRL and GAL for homotypic seeding as lag time decreased significantly in the presence of $2 \%$ and $5 \%$ seeds (Fig 3b, 3e). However, $1 \%$ seed did not show any fibril formation for both PRL as well as GAL (Fig 3b, Supplementary Figure 6) even after 10 days of incubation. Fibril formation via homotypic seeding mechanism was further supported by TEM imaging and time-dependent CD spectroscopic measurements (Fig

\section{3b-c, Supplementary Figure 6).}

Similar experiments were done where various concentrations of PRL seeds were mixed with GAL monomer and GAL seeds were mixed with PRL monomer (heterotypic seeding). We hypothesized two possibilities of secondary nucleation ${ }^{55}$ - the heterotypic monomers can be recruited at the ends of the seeds and grow the amyloid fibril (elongation) or the seed surface will help in the nucleation of the heterotypic monomer ${ }^{55}$ (Fig 3a). Our data showed that GAL aggregation was accelerated with PRL seeds in a concentration-dependent manner (Fig 3d, left panel). Surprisingly, we observed no ThT fluorescence for all seed concentrations even after 10 days of incubation when GAL seeds were incubated with PRL monomers, indicating that GAL fibrils are incapable of inducing amyloid fibril formation of PRL monomers (Fig 3d, right panel). This was also evident with CD spectroscopic measurements (Supplementary Fig 7). Interestingly, no aggregation was observed when PRL/GAL monomer was incubated in presence of different percentages of PRL-GAL mixed fibril seeds $(1 \%, 2 \%$, and $5 \%)$ as confirmed by CD spectroscopy, ThT fluorescence, and TEM imaging (Supplementary Fig 8-9). This suggests that seeding (both homo and hetero) event is very 
specific for the life cycle of PRL/GAL amyloid formation in SG. Important to note that these homotypic and heterotypic seeding studies were done in the absence of any helper molecules of GAGs. When lag times were compared, the heterotypic seeding rate was significantly higher than homotypic seeding for GAL aggregation suggesting surface-mediated secondary nucleation might be triggering GAL aggregation in the presence of PRL seeds ${ }^{56}$ (Fig 3e).

To further understand whether PRL seeds engage the GAL monomer for secondary nucleation $^{27}$ where elongation and/or surface-mediated aggregation could happen ${ }^{55}$, fibril diameters of GAL (formed by PRL cross-seeding) were analyzed from the TEM images (Fig 3f). If the elongation/fragmentation mechanism is occurring, GAL will form PRL-like fibrils. If PRL seeds engage GAL monomer for surface-mediated secondary nucleation, GAL will essentially form GAL-like fibrils. Intriguingly, analysis of frequency distribution of the GAL fibril diameters indicated that the distribution of diameter of PRL seeded GAL fibrils was very similar to that of GAL-Hep fibrils (Fig 3g, left panel). The median and mean fibril diameter analysis also suggests that GAL fibril formed in presence of PRL seed (median and mean diameter $12.35 \mathrm{~nm}$ ) is very similar compared to GAL fibrils formed in presence of GAL seed (median and mean diameter $12 \mathrm{~nm}$ ) as well as GAL fibrils formed in presence of heparin (median and mean diameter $11.20 \mathrm{~nm}$ ) (Fig 3g, right panel, Supplementary Figure 6). This means that the incorporation of GAL monomers does not happen to the PRL fibril end (elongation) $)^{25,51,57,58}$, rather, GAL monomers use PRL seeds as surface and form amyloid fibrils via secondary (or surface) nucleation ${ }^{26,52,53,56}$ mechanism. This was further confirmed with the FTIR study, which suggested that GAL-Hep and GAL in the presence of PRL seed possessed identical FTIR spectral signature, which was substantially different from the PRL fibril spectrum (Fig 3h).

\section{Specific interactions of PRL and GAL leading to amyloid aggregation}

Next, we wanted to further investigate if interactions leading to co-aggregation and amyloid formation of PRL and GAL are specific to themselves. To do this, we co-incubated PRL with adrenocorticotropic hormone (ACTH) (Supplementary Table 1) as this hormone is of similar length to GAL and does not form amyloid by itself ${ }^{13,59}$. Similarly, GAL was also incubated with growth hormone (GH) (Supplementary Table 1), a hormone structurally and functionally related to $\mathrm{PRL}^{60,61}$. ThT aggregation kinetics and CD spectroscopy were performed at the beginning of the aggregation (day 0 ) and after 15 days of incubation for both PRL-ACTH and GAL-GH. Our data showed negligible ThT fluorescence for both PRL- 
ACTH and GAL-GH even after 15 days (Fig 4a) suggesting no co-aggregation. This observation was consistent with no structural conversion observed in CD and TEM, where the PRL-ACTH and GH-GAL mixtures were devoid of any fibrils (Fig 4b, Supplementary Figure 10). Overall, our observations suggest that interaction and co-aggregation/amyloid formation by PRL and GAL are specific and are mutually beneficial for the storage of these hormones in SGs. This specific co-aggregation of PRL and GAL could be due to their favorable interaction when the monomeric hormone is mixed together. This is further evident from the surface plasmon resonance (SPR) study, where GAL monomers were immobilized on a CM-5 chip and a range of concentrations of PRL monomeric protein was passed over it. We observed a significant increase in the response unit (RU) indicating binding of PRL to GAL (Fig 4c). The relative dissociation constant $\left(\mathrm{K}_{\mathrm{D}}\right)$ was calculated to be $4.1 \times 10^{-7} \mathrm{M}$, which indicates the binding of PRL with GAL (Fig 4d). In comparison, we observed no significant binding when ACTH was passed through immobilized PRL or when GH was passed through immobilized GAL (Fig 4c). This suggests that PRL monomers can readily bind GAL monomers, possibly contributing to their initial interaction that eventually drives the synergistic aggregation and amyloid formation.

To understand the mechanism of PRL and GAL interactions at the atomic level, docking, and molecular dynamics (MD) simulation studies were performed. GAL was docked at two different regions of PRL that showed high TANGO score (Fig. 1b,c). Thus, two sets of PRLGAL complexes were generated (a) Set 1: GAL was docked near residues 18-28 of PRL and (b) Set 2: GAL was docked near residues 80-88 of PRL. The lowest energy docked complexes from each set, named complex-1 and complex-2 respectively, were obtained (Supplementary Figure 11). Both these complexes were then subjected to independent 250ns long MD simulations to examine their stability. Since the choice of force fields may play an important role in the MD simulation results ${ }^{62}$, we performed MD simulations with two different force fields - GROMOS 53a6 force field ${ }^{63}$ and Amber ff99SB force field ${ }^{64}$. As controls, we have also simulated individual PRL and GAL proteins. We observed that the PRL and GAL alone did not show any noticeable structural changes (Supplementary Figure 11) during simulation time. 
bioRxiv preprint doi: https://doi org/10.1101/2021.08.31.458467; this version posted September 1, 2021. The copyright holder for this preprint (which was not certified by peer review) is the author/funder, who has granted bioRxiv a license to display the preprint in perpetuity. It is made available under aCC-BY-NC-ND 4.0 International license.
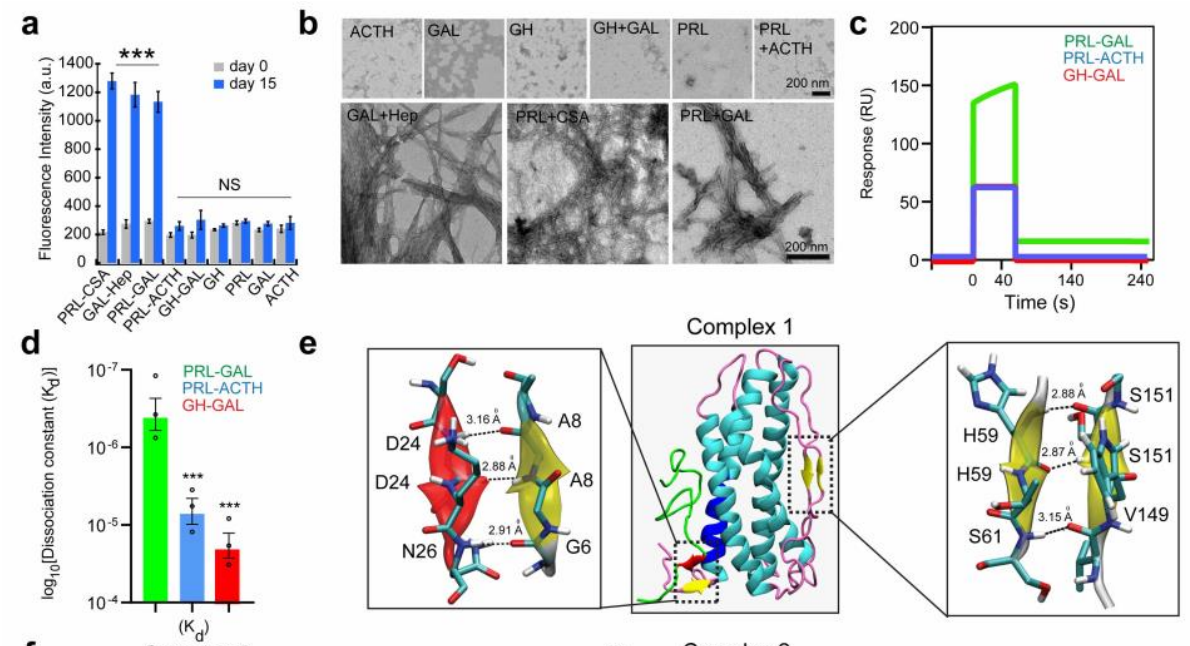

f

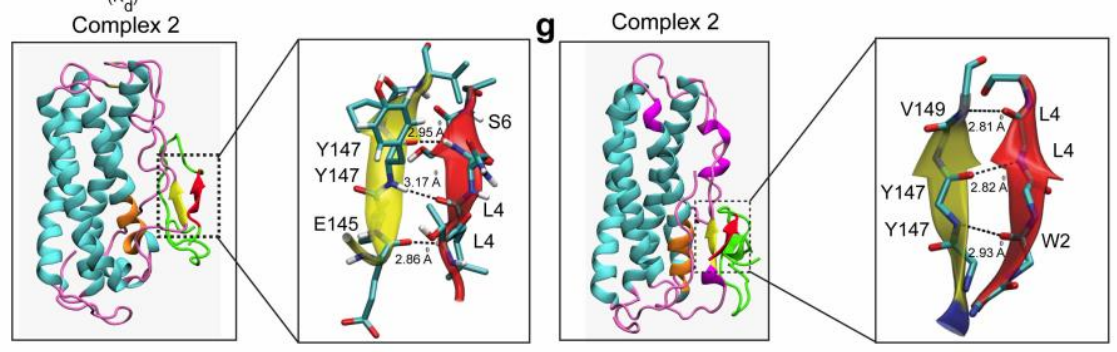

Fig 4: Specific interaction drives co-aggregation of PRL and GAL:

a. Comparative ThT fluorescence showing amyloid formation by different pairs of hormones at days 0 and 15. PRL-CSA, GAL-Hep, PRL-GAL showed the highest ThT fluorescence signals after 15 days of incubation. Values represent mean \pm SEM for $n=3$ independent experiments. The statistical significance is calculated between day 0 and day 15 for each sample using a t-test. b. The morphology observed under TEM for various hormones and the mixture of hormone samples are shown (after 15 days of incubation). Amorphous structures are seen for PRL-ACTH and GAL-GH; whereas PRLGAL, PRL-CSA, GAL-Hep showed fibrillar morphology similar to amyloids. The experiment is performed three times with similar observations. c. Surface Plasmon Resonance (SPR) spectra showing strong binding of PRL on immobilized GAL compared to other pairs of hormones. d. The dissociation constant $\left(\mathrm{K}_{\mathrm{d}}\right)$ of PRL to GAL showing strong interaction between PRL and GAL for their co-aggregation and co-storage. The experiments are performed three times with similar results. Values represent mean \pm SEM $(* * * p \leq 0.001, * p \leq 0.05)$. e. Snapshot from in silico analysis (MD simulation) of PRL-GAL complex-1 using GROMOS 53a6 force field (when GAL is docked near residues 18-28 of PRL). f. Snapshot showing MD simulation of PRL-GAL complex-2 (when GAL is docked near residues 80-88 of PRL). Complex-1 induced the formation of an antiparallel $\beta$-sheet at the PRL-GAL interface (6-8 PRL \& 24-26 GAL) and also an intra-molecular parallel $\beta$-sheet in PRL itself (59-61 PRL \& 149-151 PRL). Complex-2 shows the formation of a parallel $\beta$-sheet constituted by the $\beta$-strand from PRL and GAL (145-147 PRL and 4-6 GAL). g. Snapshot showing MD simulation of complex-2 using amber ff99SB force field shows the appearance of parallel $\beta$-sheet at 147-149 residue of PRL and 2-4 residue of GAL. The snap-shot of complex-1 is included in Supplementary Figure 11.

On the contrary, the PRL-GAL complexes exhibited significant conformational changes upon binding to each other, in both complexes (Fig 4e-g). The respective structures of complex-1 and complex-2 at the end of the MD simulation using GROMOS force field showed the 
structural transition from unstructured region to $\beta$-strand in both PRL and GAL with the emergence of a parallel or anti-parallel $\beta$-sheet at the protein-protein interface (Fig 4e,f). The interaction of GAL in complex-1 induced the formation of an antiparallel $\beta$-sheet at the PRLGAL interface (residues 6-8 of PRL, residues 24-26 in GAL) and also an intra-molecular parallel $\beta$-sheet in PRL itself (PRL residues 149-151 and 58-60) (Fig 4e). In complex-2, GAL induced the formation of parallel $\beta$-sheet at PRL-GAL interface (residues 145-147 of PRL, residues 4-6 of GAL) (Fig 4f). From the MD simulations using the Amber force field, however, the complex-1 did not show any notable structural changes, except that the terminal loops in PRL wrap around the existing secondary structures for higher stability (Supplementary Fig 11). However, the PRL-GAL interactions in complex-2 resemble very well with the results from the GROMOS force field exhibiting a parallel $\beta$-sheet constituted by the $\beta$-strand from PRL and GAL proteins (Fig $\mathbf{4 g}$ ). These results corroborate very well with our experimental data that suggested the formation of amyloids when PRL and GAL were co-aggregated. The residues involved in the formation of this $\beta$-sheet in complex- 2 were PRL residues 147-149 and GAL residues 2-4 in the Amber ff99SB force field (Fig 4g). Thus, irrespective of the force field used, our MD simulation results convincingly show that the coaggregation of PRL and GAL induce the formation of $\beta$-sheet at the protein interface.

\section{Release of functional PRL and GAL from PRL-GAL amyloids}

Protein/peptide misfolding and aggregation are known to lead to irreversible amyloid formation, which is stable and not readily disassemble to monomer. However, many studies recently showed the release of monomers and oligomers from disease-associated amyloids ${ }^{65,66}$. In contrast, amyloid formation related to SG biogenesis is reversible and should be able to release functional monomers in the extracellular space for their function ${ }^{13,14,17}$. To address whether any functional advantage of co-aggregation over homotypic aggregation by PRL and GAL, we determined the relative monomer release capability of PRL, GAL, and PRL-GAL co-amyloids, preformed fibrils of PRL (in the presence of CSA), GAL (in the presence of Hep) using dialysis method ${ }^{13,14,17}$. The concentration of released monomers (if any) in the dialysate was measured by UV-Vis spectroscopy at different time points. Intriguingly, we found that PRL-CSA, GAL-Hep, and PRL-GAL amyloids could indeed release monomers with time (Fig 5a). Interestingly, the amyloid fibrils of PRL and GAL formed in presence of GAGs (CSA and Hep, respectively) released monomeric hormones in a slow and sustained manner upon dilution in 10 mM Tris-HCl, pH 7.4 (Fig 5a). 

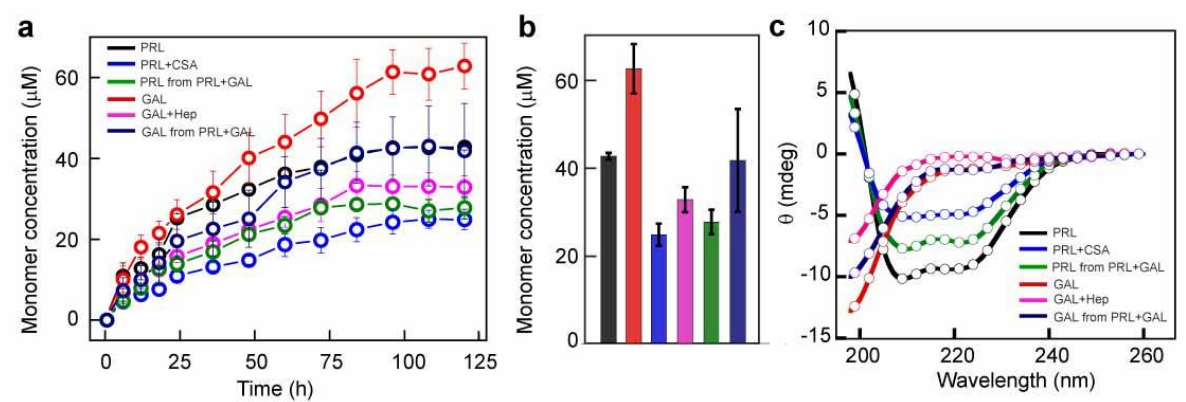

d
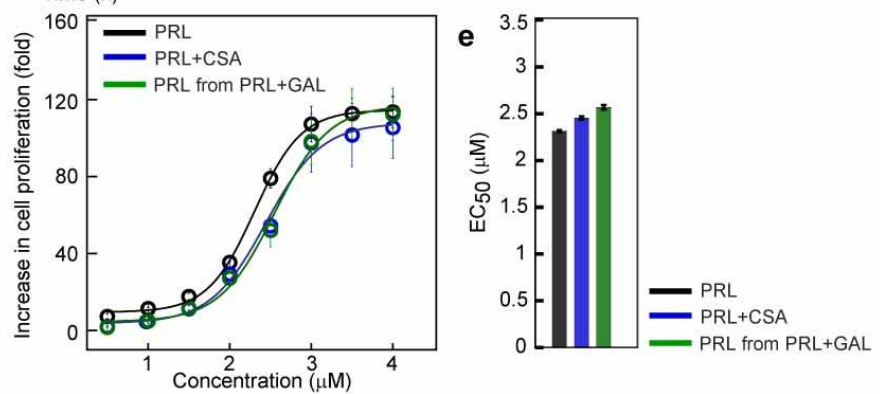

\section{Fig 5: Monomer release from PRL and GAL amyloid}

a. The kinetics of monomer release from various amyloids showing the continuous release of monomeric hormones. The experiment is performed three times with similar results. Values represent mean \pm SEM for $n=3$ independent experiments. b. Saturation concentrations of different released monomers from fibrils along with the monomeric controls are shown. Values represent mean \pm SEM for $n=3$ independent experiments. c. The secondary structure of released monomers showing their corresponding native secondary structures as confirmed by the $\mathrm{CD}$. d. $\mathrm{Nb} 2$ cell proliferation study showing biological activity of released PRL from either PRL+CSA or PRL+GAL fibrils. Freshly dissolved protein was used as a control. Values represent mean \pm SEM for $n=3$ independent experiments. e. $\mathrm{EC}_{50}$ values showing the released PRL monomers have similar bioactivity compared to freshly dissolved monomeric PRL. Values represent mean \pm SEM for $n=3$ independent experiments.

However, the release of monomeric PRL and GAL hormones from the co-aggregated PRLGAL fibril was faster compared to the PRL and GAL released from their GAGs mediated fibrils. This was confirmed by their release profile as well as saturation concentrations (Fig 5a-b). CD spectroscopy of the dialysate showed that the released PRL monomer retained its native conformation $(\mathbf{F i g} \mathbf{5 c})$.

Next, we performed the cell proliferation assay with the released PRL monomer to check for the retention of bioactivity of PRL. We used the $\mathrm{Nb} 2$ cell line for this study ${ }^{67}$. $\mathrm{Nb} 2$ cells are rat lymphoma cells with significant expression of PRL receptors on the cell surface 67,68 . These cells require PRL for proliferation or mitogenesis ${ }^{68,69}$. We observed that released PRL monomers obtained from PRL-CSA fibrils and PRL-GAL fibrils were functional as they can induce cell proliferation in a dose/concentration-dependent manner (Fig 5d). The $\mathrm{EC}_{50}$ values 
of the PRL released from PRL fibrils are similar as of freshly prepared PRL monomer, suggesting no difference in their functionality (Fig 5e).

\section{Discussion}

Amyloids are ordered protein aggregates comprised of cross- $\beta$-sheet motifs where $\beta$-sheets are parallel, and individual $\beta$-strands are perpendicular to the fibril axis ${ }^{47,70}$. Despite their association with diseases, amyloids are also known to be involved in the native functions of host organisms including mammals ${ }^{71,72}$. Interestingly, the synergistic amyloid formation through co-aggregation and cross seeding by heterologous proteins/peptides is evident in the disease-associated amyloid formation such as $\alpha$-Synuclein-Tau ${ }^{73,74}, \alpha$-Synuclein-amyloid$\beta^{28,75}$. However, co-aggregation and cross seeding associated with functional amyloids are still elusive although this is of high functional relevance as many hormones are co-stored and co-released from secretory granules. Here, we explored the synergistic aggregation and amyloid formation by two human hormones prolactin (PRL) and galanin (GAL), with relevance to SG formation. Although $23 \mathrm{kDa} \mathrm{PRL}^{41,42}$ with mostly helical structure have no resemblance of sequence, length, and structure with unstructured $3.1 \mathrm{kDa} \mathrm{GAL}^{43,44}$, it was suggested that both of these hormones are co-stored in lactotrophs of anterior pituitary female rats $^{38}$ and their release are also modulated by the same secretagogues ${ }^{35,36,40}$. Their co-storage and co-release suggest that they might aggregate together to form amyloid within the same secretory granules for storage. Indeed, the immunofluorescence study with anterior pituitary tissue showed not only both PRL and GAL do colocalize together but are also in the amyloid form as suggested by OC and ThioS staining (Fig 1 and Supplementary Fig 1). Interestingly when PRL and GAL were co-incubated with a 1:1 or higher molar ratio in vitro, they coaggregated synergistically to form amyloid fibrils in conditions similar to their storage in SGs without the requirement of any helper ${ }^{76,77}$ molecules.

PRL and GAL co-aggregation can happen where both PRL and GAL can promote the aggregation of each other but also form individual homotypic fibrils (Fig 2). Further, one of them might have the conformational advantage to form amyloids and other proteins/peptides can simply adhere to it (Fig 2). Also, PRL-GAL can be incorporated within the same fibrils or filaments (Fig 2). When immuno-EM was done with the secondary antibodies of differentsized gold nanoparticles, we observed both PRL and GAL are present within the same fibrils suggesting they might interact together to form hybrid fibrils (Fig 2). The incorporation of heterogenous protein to make hybrid fibrils is also shown in $\alpha$-Synuclein ( $\alpha$-syn) and Tau and other fibril formtion ${ }^{28,73-75}$. The co-aggregation without GAGs where the individual hormone 
is unable to form amyloids further supports that in GAGs deficient condition, PRL and GAL might more prefer to form mixed amyloid for their storage. However, their co-aggregation does not preclude the possibility of individual storage of each hormone in the different SGs as both PRL and GAL formed amyloids in presence of respective helper GAGs molecules. Individual hormone aggregation can be seeded with their respective fibrils seeds as shown for PRL and GAL even in absence of GAGs, supporting the fact that amyloid fibrils formation might be very productive and formed autocatalytic manner inside the secretory cells.

Most often homologous seeding occurs for amyloid aggregation and decreases the lag time for aggregation ${ }^{20,21}$. Heterogeneous seeds also provide template/surface to protein/peptide for their aggregation, but not always cross seeding happens as barriers exist between two different protein/peptides ${ }^{25,52,53}$. It was shown that sequence similarity between two amyloidogenic proteins is crucial for cross seeding capability ${ }^{78}$. However, the ability of cross seeding also depends on the conformation of the seed and its compatibility with conformation or sequence of the monomeric protein, which creates different cross seeding barrier ${ }^{25,51,79}$ (also species barrier for prion diseases).

Our data showed PRL amyloid fibrils can cross-seed GAL monomers to form amyloid fibrils. Strikingly, preformed GAL fibrils were not able to cross seed PRL monomers suggesting specificity and regulation in functional amyloid formation (Fig 3). Such unidirectional, as well as bidirectional seeding between amyloid proteins, are also evident. For example, cross seeding between $A \beta_{42}$ and hIAPP, where the $A \beta_{42}$ seeds can cross seed the hIAPP to promote aggregation but hIAPP seed was unable to induce aggregation to $A \beta_{42}$ monomers ${ }^{80}$, suggesting the unidirectional cross seeding. In contrast, $\alpha$-syn seeds speed up the aggregation of Tau and Tau seeds also accelerated the aggregation of $\alpha$-syn suggesting the bidirectional cross seeding ${ }^{73,74}$.

Since PRL has less propensity to form amyloid due to higher structural stability, GAL seeds are not able to provide neither surface nor structural compatibility for PRL for cross seeding. This might suggest that tight regulation of co-species aggregation ensures the right amount of storage of PRL and GAL for secretory storage with proper ratio. Moreover, GAL is a highly disordered peptide, which might easily bind to the PRL amyloid surface nonspecifically and increase its stability and aggregates. Further PRL amyloid-prone sequence could also be sequestered inside the helical structure, which might not be compatible with either surface and/or amyloid core structure of GAL to mediate the cross-seeding. Previously it was shown that cross seeding of K18 and K19 of Tau isoforms, K19 fibrils can cross seed K18 through the catalytic motif of R3, whereas K18 fibrils with the catalytic center as R2 is unable to seed 
$\mathrm{K} 19^{81}$ (lacking R2 motif). The difference in seeding could also be due to the relative tendency of amyloid fibril formation by GAL and PRL. We propose that PRL fibrils seeding GAL aggregation is not due to elongation and fragmentation (which require specific interaction) as PRL cross- $\beta$ spine might not be assessable for GAL. We previously proposed that a small segment of GH (structurally similar to PRL) might engage for fibrils formation ${ }^{14}$ and other structural domains might be surrounded to the cross- $\beta$ spine as proposed for RNaseA fibrils model $^{82}$. We believe due to the unstructured peptide of GAL, it could easily adhere to the surface of PRL amyloid seeds and proceeds for surface-mediated secondary nucleation. This is further supported as the structure of PRL seeded GAL fibril showed similar fibrils morphology and secondary structure as of GAL fibrils formed alone (Fig 3). This is a typical property of surface-mediated secondary nucleation that produce fibrils ${ }^{56}$. Further the PRL seeding to GAL fibril formation was more efficient as compared to homo-seeding of GAL further support surface mediated secondary nucleation ${ }^{53,56}$ (Fig 3).

We hypothesized that specific interaction between GAL and PRL synergistically facilitates their aggregation into amyloid. This is further evident from the SPR analysis showing strong interaction and direct binding between the monomeric forms of PRL and GAL in contrast to the other hormone pair of PRL-ACTH or GH-GAL (Fig 4). This PRL-GAL interaction and conformation transition are further supported using in silico study, which showed interaction of the PRL N-terminal loop and GAL, where GAL promotes the conformational transition of the N-terminus of PRL into $\beta$ structure (Fig 4). The interaction of PRL and GAL is mandatory for their aggregation, as amyloids were not formed when PRL and GAL were incubated alone. 


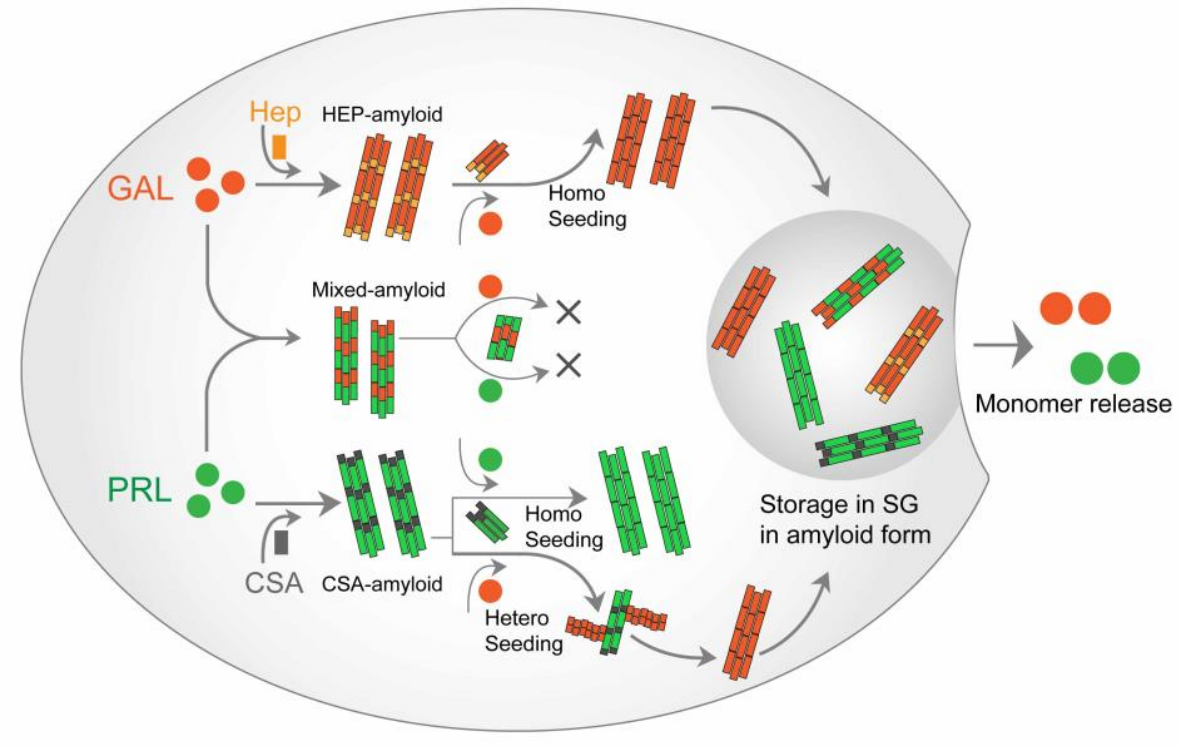

Figure 6: PRL-GAL homo and hetero amyloid life cycle for secretory granule

PRL and GAL form the amyloid fibrils in the presence of specific GAGs (CSA and Hep, respectively), which can be auto-catalytically amplified by their respective seeding with preformed fibrils. This seeding however does not require any GAGs. PRL-GAL also synergistically co-aggregate to form hybrid amyloid fibrils, which are not capable of seeding either to PRL or GAL. These amyloid fibril species can together or individually reconstitute the SGs of PRL-GA storage, which can release functional PRL and GAL into the extracellular space.

The observed co-aggregation and cross-seeding may not only give the advantage for both PRL and GAL to be stored as amyloids (and nonrequirement of helper molecules) but also may help for their subsequent release after secretion. This is supported by the fact that the monomer release from PRL-CSA amyloid and GAL-Hep amyloid was much slower compared to the release of mixed amyloid of PRL-GAL (Fig 5). Further, our study suggests that nature has optimized hormone storage in such a way that one hormone can be stored in different ways in different secretory granules and the release of these hormones also depends on the type of amyloid aggregate, which is stored in the SG (Fig 6). The present study suggests that contrary to disease-associated co-aggregation, which promotes the spread of the disease, co-aggregation of hormones is specific and functional in the SGs. Co-aggregation and amyloid formation of these structurally dissimilar hormones indicate the relevance of amyloid as a crucial aspect of cellular sorting and storage in SG biogenesis. 


\section{Materials and Methods}

\section{Chemicals and Reagents}

The peptide GAL was purchased from USV Limited (Mumbai, India) by custom synthesis and other chemicals were obtained from Sigma Chemicals or other sources with the highest purity available.

\section{Expression and purification of human Prolactin (PRL)}

PRL was expressed as per the protocol reported ${ }^{83}$ with little modification. The human PRL plasmid was obtained as a kind gift from Prof. Dannies and Prof. Hodsdon from Yale University. BL21 (DE3) E. coli cells were transformed with the hPRL gene encoded in the pT7L plasmid and were made to grow in terrific broth (TB) followed by IPTG induction for 4 hours. After harvesting the cells at $8000 \mathrm{rpm}$ for 20 minutes, it was dissolved in $20 \mathrm{mM}$ Tris$\mathrm{HCl}, \mathrm{pH} 8.0$ (with added protease inhibitor cocktail, Roche). After that, the cells were sonicated ( 2 seconds on, 1 second off at 50\% amplitude) for 20 minutes to complete celllysis, which was then centrifuged at $15000 \mathrm{rpm}$ for half an hour to recover the inclusion bodies (IB). The cell pellet containing IBs was subsequently washed two times with $0.5 \%$ triton-X and then it was dissolved in $8(\mathrm{M})$ urea with $2 \%(\mathrm{v} / \mathrm{v}) \beta$-mercaptoethanol. The solution was then dialyzed against $20 \mathrm{mM}$ Tris- $\mathrm{HCl}, \mathrm{pH} 8.0$ so that the PRL protein refolds to its native state. After dialysis, the protein solution was again centrifuged at $15000 \mathrm{rpm}$ for 1 hour and was loaded in an anion exchange column (Resource Q, GE healthcare) through an AKTA purifier FPLC system (Cytiva). The protein was eluted through $1(\mathrm{M}) \mathrm{NaCl}$ gradient and subsequently lyophilized after snap-freezing in liquid nitrogen. Size exclusion profile (SEC) suggested the protein is monomeric in nature and the purity of the protein is further checked by SDS-PAGE and MALDI-TOF spectrometry. CD spectroscopy was also performed to confirm that the purified PRL has refolded to its native helical conformation.

\section{Aggregation of PRL and GAL in the presence of GAGs}

PRL was dissolved in MQ water and buffer exchanged to $20 \mathrm{mM}$ phosphate buffer with 100 $\mathrm{mM} \mathrm{NaCl}, \mathrm{pH}$ 6.0, 0.01\% sodium azide using $10 \mathrm{kDa}$ mini dialysis units (Thermo Scientific 
Slide-A-Lyzer). $5 \mathrm{mM}$ solution of CSA (Sigma, USA) was prepared in the same buffer, and appropriately mixed with PRL to obtain an ultimate concentration of $400 \mu \mathrm{M}$ for both PRL and CSA. Similarly, for GAL aggregation in the presence of Hep, GAL peptide was dissolved in $20 \mathrm{mM}$ phosphate buffer containing $100 \mathrm{mM} \mathrm{NaCl}, \mathrm{pH}$ 6.0, 0.01\% sodium azide to obtain a concentration of $500 \mu \mathrm{M}$. Hep solution from a stock of $5 \mathrm{mM}$ (made in the same buffer) was then mixed with GAL solution to obtain an ultimate concentration of $400 \mu \mathrm{M}$ for both GAL and Hep. These tubes containing PRL and GAL of various mixtures were kept into an Echo Thermmodel RT11 rotating mixture with a speed of $50 \mathrm{rpm}$ for 15 days inside a $37^{\circ} \mathrm{C}$ incubator. As a control, $400 \mu \mathrm{M}$ PRL and GAL alone in the same buffer, was also incubated in a similar condition. The secondary structural transition was monitored by CD spectroscopy and amyloid formation by ThT binding assay at various time points. Finally, Congo red (CR) binding studies and TEM imaging was used to confirm amyloid fibril formation.

\section{Co-aggregation study of PRL and GAL}

For the co-aggregation study, PRL was dissolved in Milli-Q water and buffer exchanged to $20 \mathrm{mM}$ phosphate buffer with $100 \mathrm{mM} \mathrm{NaCl}, \mathrm{pH}$ 6.0, 0.01\% sodium azide using $10 \mathrm{kDa}$ mini dialysis units (final concentration was $800 \mu \mathrm{M}$ ) (Thermo Scientific Slide-A-Lyzer). GAL was also dissolved in the same buffer to obtain an $800 \mu \mathrm{M}$ solution. After that, each of the solutions was mixed to obtain $400 \mu \mathrm{M}$ of PRL-GAL mix and was incubated with slight agitation at $37^{\circ} \mathrm{C}$ for two weeks. $400 \mu \mathrm{M}$ of each PRL and GAL was incubated alone as controls. For co-aggregation of PRL and GAL with other hormones, separate solutions of PRL, GAL, GH, and ACTH were freshly dissolved and prepared in identical solution condition as above. $200 \mu \mathrm{l}$ of each solution were mixed to obtain $400 \mu \mathrm{M}$ each of PRLACTH and GAL-GH mixture and was incubated with slight agitation at $37{ }^{\circ} \mathrm{C}$ for two weeks. $400 \mu \mathrm{M}$ of each of PRL, GAL, GH and ACTH were also incubated alone as controls. The secondary structural transition and amyloid formation of the incubated solutions were 
monitored by CD spectroscopy and ThT binding assay during various time points. After 15 days of incubation, the morphology of the incubated samples was analyzed by TEM.

\section{Circular Dichroism spectroscopy (CD)}

For CD measurement, protein/peptide aliquots were diluted in $20 \mathrm{mM}$ phosphate buffer with $100 \mathrm{mM} \mathrm{NaCl}, \mathrm{pH} 6.0,0.01 \%$ sodium azide to $200 \mu \mathrm{l}$ and the final concentration protein/peptide was $10 \mu \mathrm{M}$. CD spectra were taken using a JASCO 810 instrument where the sample was loaded in a quartz cell of $0.1 \mathrm{~cm}$ path length (Hellma, Forest Hills, NY). Spectra were collected at $198-260 \mathrm{~nm}$ wavelength (far UV) at $25^{\circ} \mathrm{C}$. Raw data was processed by smoothening, as per the manufacturer's instructions. Three independent experiments were performed with each sample.

\section{Thioflavin T (ThT) Binding Assay}

To measure ThT binding, PRL and GAL solutions were diluted in the same buffer into $200 \mu \mathrm{l}$ such that final concentration of each sample was $10 \mu \mathrm{M} .4 \mu \mathrm{l}$ of $1 \mathrm{mM}$ ThT prepared in 20 $\mathrm{mM}$ Tris-HCl buffer, $\mathrm{pH} 8.0$ was added into each sample. ThT fluorescence was probed after the immediate addition of ThT. The fluorescence experiment was carried out in Shimadzu RF5301 PC, with excitation wavelength at $450 \mathrm{~nm}$ and emission wavelength from 460-500 $\mathrm{nm}$. For measuring both excitation and emission, the slit width was kept at $5 \mathrm{~nm}$. Three independent experiments were performed for each sample.

The lag time $\left(\mathrm{t}_{\text {lag }}\right)$ was calculated as per the published protocol $^{84}$ :

$\mathrm{y}=\mathrm{y}_{0}+\left(\mathrm{y}_{\max }-\mathrm{y}_{0}\right) /\left(1+\mathrm{e}^{-\mathrm{k}(\mathrm{t}-\mathrm{t} 1 / 2)}\right)$

here $\mathrm{y}$ is the ThT fluorescence at any particular time point,

$\mathrm{y}_{\max }$ is the maximum ThT fluorescence observed and $\mathrm{y}_{0}$ is the ThT fluorescence at $\mathrm{t}_{0}$ (initial time) and $t_{\text {lag }}$ was defined by

as $\mathrm{t}_{\mathrm{lag}}=\mathrm{t} 1 / 2-2 / \mathrm{k}$ 


\section{Congo red (CR) binding}

A $5 \mu \mathrm{l}$ aliquot of protein/peptide sample was added into $80 \mu \mathrm{l}$ of $5 \mathrm{mM}$ potassium phosphate buffer containing $10 \%$ ethanol. $100 \mu \mathrm{M}$ CR solutions were prepared in $5 \mathrm{mM}$ phosphate (containing $10 \%$ ethanol) and $15 \mu \mathrm{l}$ of the solution was added to the sample. After incubating for $15 \mathrm{~min}$ in dark, absorption spectra were taken from 300-700 nm (JASCO V-650 spectrophotometer). For control CR solution without protein was also measured. Three independent experiments were performed for each sample.

\section{CR birefringence study}

Protein fibrils were obtained by ultracentrifuging the fibril solution at 95,000 rpm for 1 hour followed by washing with Milli-Q water. The fibrils were mixed in $100 \mu 1$ of alkaline sodium chloride solution for $20 \mathrm{~min}$ with vortexing, to ensure uniform mixing of all fibrils in solution. The mixture was further centrifuged and pellet fractions were stained with alkaline CR solution for 20 minutes with vortexing. After that, mixtures were again centrifuged at 95,000 rpm for 1 hour, and pellets were washed two times by $500 \mu \mathrm{l}$ of $20 \%$ ethanol. The pellets were then resuspended in PBS and spotted onto glass slides and subjected to airdrying at room temperature. The slides were observed using a microscope (Olympus SZ61 stereo zoom) attached with two polarizers and a camera.

\section{Immunoelectron microscopy}

$10 \mu 1$ of PRL-GAL or PRL-CSA fibril was spotted onto the TEM grid. $10 \mu 1$ of rabbit antiPRL antibody (1:10) and/or mouse anti-GAL antibody (1:10) in PBS was added to the fibrils and was incubated for $1 \mathrm{~h}$. The excess antibody was removed using filter paper. The grid was subsequently washed thrice with autoclaved MQ water. Anti-mouse secondary antibody conjugated with $10 \mathrm{~nm}$ gold particles (1:200) and/or anti-rabbit secondary antibody conjugated with $5 \mathrm{~nm}$ gold particles (1:200) was added to the grid and incubated for $30 \mathrm{~min}$. The grid was then washed thrice with MQ water, followed by staining with $1 \%$ uranyl 
formate for $5 \mathrm{~min}$ and it was imaged using TEM (CM200, Netherland), and analyzed using KEEN view software.

\section{Transmission electron microscopy (EM)}

The protein/peptide sample was diluted in Milli-Q water to $\sim 60 \mu \mathrm{M}$. Then, the samples were spotted on a carbon-coated, glow-discharged Formvar grid (Electron Microscopy Sciences, Fort Washington, PA) and were kept for incubation for $5 \mathrm{~min}$. The grids were further washed with Milli-Q water and were stained with a $1 \%(\mathrm{w} / \mathrm{v})$ uranyl formate solution. TEM imaging was done using FEITecnai $\mathrm{G}^{2} 12$ electron microscope at either $120 \mathrm{kV}$ or $200 \mathrm{kV}$ with nominal magnifications in the range of $26,000-60,000$. Images were collected by using the SIS Megaview III imaging system. Independent experiments were carried out thrice for each sample.

\section{X-ray fibril diffraction}

PRL/GAL fibrils were isolated by ultracentrifugation as mentioned earlier and were loaded into a clean $0.7 \mathrm{~mm}$ capillary. The samples in capillary were dried overnight under vacuum. The whole capillary with dried protein was placed in the path of X-ray beam. The dried film of protein was placed in an X-ray beam at $200 \mathrm{~K}$ for $120 \mathrm{~s}$ exposure. The resulting images were collected using a Rigaku R-Axis IV++ detector (Rigaku, Japan) kept on a rotating anode. The distance between the sample to the detector was $200 \mathrm{~mm}$ and the image files were analyzed and processed using Adxv software.

\section{FTIR spectroscopy}

For FTIR spectroscopy, isolated fibrils or monomers were spotted onto a thin $\mathrm{KBr}$ pellet and were subjected to dry under an IR lamp. Then the spectrum was collected using a Bruker VERTEX 80 spectrometer attached with a DTGS detector at the frequency range of 1800$1500 \mathrm{~cm}^{-1}$, corresponding to the amide I stretching frequency, with a resolution limit of $4 \mathrm{~cm}^{-}$ 1. The recorded spectrum was deconvoluted at the frequency range $1700-1600 \mathrm{~cm}^{-1}$, using 
bioRxiv preprint doi: https://doi.org/10.1101/2021.08.31.458467; this version posted September 1, 2021. The copyright holder for this preprint (which was not certified by peer review) is the author/funder, who has granted bioRxiv a license to display the preprint in perpetuity. It is made available under aCC-BY-NC-ND 4.0 International license.

Fourier Self Deconvolution (FSD) method and the deconvoluted spectrum was fitted using the Lorentzian curve fitting method using OPUS-65 software (Bruker, Germany) according to the manufacturer's instructions. Independent sets were performed thrice for each sample.

\section{Seeding and cross-seeding by different fibril-seed}

Amyloid formation by PRL/GAL was confirmed by ThT binding and TEM imaging. After that, various fibrils were collected seperately via ultra-centrifugation, and each fibril sample was suspended in $20 \mathrm{mM}$ phosphate buffer, $\mathrm{pH} 6.0,100 \mathrm{mM} \mathrm{NaCl}, 0.01 \%$ sodium azide. These fibrils were then subjected to sonication (03 seconds“on" and 01 second "off" at $20 \%$ amplitude) for 10 minutes to obtain preformed fibril seeds, which were mixed in the respective monomeric protein $(1 \%, 2 \%$ and $5 \%(\mathrm{v} / \mathrm{v}))$ for homo seeding or to the other protein for cross seeding. The time-dependent aggregation was probed by ThT binding and CD spectroscopy.

\section{Surface plasmon resonance (SPR) spectroscopy analysis of PRL-GAL interaction}

GAL was immobilized on to Biacore CM5 sensor chip (GE Healthcare) via amine coupling. To do that, $300 \mu \mathrm{g} / \mathrm{ml}$ of GAL solution was made in $50 \mathrm{mM}$ sodium acetate buffer, $\mathrm{pH} 5$, and was injected at a rate of $10 \mu \mathrm{l} / \mathrm{min}$ for $720 \mathrm{~s}$ to achieve a response unit (RU) of $1252 \mathrm{RU}$. PRL protein was dissolved in $20 \mathrm{mM}$ phosphate buffer containing $100 \mathrm{mM} \mathrm{NaCl}, \mathrm{pH} 6.0$ to obtain $1 \mathrm{mM}$ stock solution and was used for preparing 7.8, 15.6, 31.2, 62.4, 125, 250, and $500 \mu \mathrm{M}$ dilutions. These solutions were then injected over the immobilized GAL at a flow rate of $45 \mu \mathrm{l} / \mathrm{min}$ for $60 \mathrm{~s}$. The dissociation was initiated at a flow rate of $30 \mu 1 / \mathrm{min}$, and the signal was recorded for $300 \mathrm{~s}$. The chip was further regenerated using a $10 \mathrm{mMNaOH}$ solution. ACTH and GH protein were used to examine the binding with PRL and GAL, respectively using a CM5 chip. For immobilization, $500 \mu \mathrm{g} / \mathrm{ml}$ of ACTH solution was made in $50 \mathrm{mM}$ sodium acetate buffer (pH4.5). For PRL immobilization, $2 \mathrm{mg} / \mathrm{ml}$ of PRL protein in $50 \mathrm{mM}$ sodium acetate buffer $(\mathrm{pH} 4)$ was used. A similar range of concentrations was used for $\mathrm{ACTH}$ and $\mathrm{GH}$ as mentioned before. All the binding experiments were performed at 37 ${ }^{\circ} \mathrm{C}$. A blank run (without analyte) was made to be subtracted from the sample response in addition to the reference surface, to rectify any instrument noise due to injections. The RU 
values obtained for each experiment were fitted using a steady-state, 2-step model and the relative dissociation constant $\left(\mathrm{K}_{\mathrm{d}}\right)$ was calculated using Biacore software.

\section{In silico study of PRL and GAL interaction and co-aggregation}

Protein-protein docking and all-atom molecular dynamics (MD) simulations were used to probe the co-aggregation propensities (if any) and resulting secondary structural transitions in PRL and GAL molecules. For this, the initial structure of PRL was obtained from PDB ID: $1 \mathrm{RW}^{41}$. Since there was no entry for GAL structure in PDB, the GAL structure was built from its primary amino acid sequence and energy minimized. Subsequently, the minimized structure was equilibrated and simulated for 300ns. Initially, GAL was docked at two amyloidogenic regions of PRL (residues 18-28) (set-1) and (residues 80-88) (set-2), which were predicted by $\mathrm{TANGO}^{45}$. The docking was performed by the protein-protein docking program, HADDOCK ${ }^{85}$. The lowest energy complexes were extracted from each set, denoted as complex-1 from set-1 and complex-2 from set-2. These two complexes were used as starting structures for the MD simulations. The first sets of simulations were performed using the AMBER16 package with the Amberff99SB force field. The LEAP module of AMBER16 was used to add the hydrogen for the heavy atoms. The complexes were then energy minimized for 2000 steps using the steepest descent and conjugate gradient algorithms. Subsequently, the structures were hydrated in a cubic periodic box extending $9 \AA$ outside the protein-protein complex on all sides with explicit water molecules. The three-site TIP3P model was chosen to describe the water molecules. The charge of each system was neutralized by placing $\mathrm{Na}^{+}$ions randomly in the simulation boxes. The systems were again minimized to prevent any bad contacts formed due to the solvation. All the systems were then equilibrated for 500ps in NVT ensemble at 300K followed by $1 \mathrm{~ns}$ in NPT ensemble at $1 \mathrm{~atm}$ of pressure. After the density and potential energy of the systems had converged, each complex was subjected to $300 \mathrm{~ns}$ of the production run. To further validate our simulations, we performed a new set of simulations using different force fields. Each complex was subjected to a 250ns simulation using the GROMOS 53a6 force field. The new sets of simulations were performed using the GROMACS package following the above-mentioned protocol. VMD tool was used for visual analysis of the trajectories. 


\section{Monomer release assay}

Amyloid fibrils of PRL and GAL formed in the presence of GAGs, and co-aggregated fibrils of PRL-GAL were harvested by ultracentrifugation at 90,000 rpm for $1 \mathrm{~h}$. The concentration of the soluble fraction (supernatant) was calculated using the absorbance at $280 \mathrm{~nm}$ (Jasco V650) and was used to determine the concentration of the pelleted fibrils. $100 \mu \mathrm{l}$ re-dissolved pellet of $400 \mu \mathrm{M}$ concentration was chosen to examine the monomer release study using the experimental setup, which has been reported previously ${ }^{14,86} .400 \mu \mathrm{M}$ PRL and GAL solutions incubated for 15 days were used as a monomer control. Briefly, For PRL monomer release, the pellet solutions (PRL, PRL-CSA, and PRL-GAL) were transferred into a modified PCR tube with a pierced hole in its cap, which was attached and sealed with a $50 \mathrm{kDa}$ molecular weight cutoff membrane (Pierce, USA). This whole setup was then placed inside a $1 \mathrm{ml}$ cryotube (Nunc, Denmark) containing $500 \mu \mathrm{l}$ of $10 \mathrm{mM}$ Tris- $\mathrm{HCl}$ buffer (pH 7.4), 0.01\% sodium azide. Meanwhile, to investigate the release of GAL monomers from the fibril of GAL-Hep, pellet solution was placed in a $10 \mathrm{kDa}$ cutoff Slide-A-Lyzer mini dialysis unit system (Pierce, USA), which was positioned onto a $1 \mathrm{ml}$ cryo-tube (Nunc, Denmark) containing $500 \mu \mathrm{l}$ of Tris- $\mathrm{HCl}$ buffer ( $\mathrm{pH} 7.4), 0.01 \%$ sodium azide. The tubes were then kept at $4^{\circ} \mathrm{C}$ to prevent evaporation of solutions. To calculate the concentration of the protein outside of the membrane, an aliquot of $100 \mu \mathrm{l}$ of the solution was pipette from the buffer outside of the dialysis membrane at different times, absorbance was measured at $280 \mathrm{~nm}$. For measuring the monomer release of PRL and GAL from PRL-GAL co-aggregates, $100 \mu \mathrm{l}$ releasing medium was taken out, and the same volume of fresh buffer was added back as volume correction (subsequent concentration correction has been done in the data). The separated $100 \mu \mathrm{l}$ releasing medium was passed through a $10 \mathrm{kDa}$ centrifugal filter unit (Amicon Ultra, Millipore). After which, the GAL monomer was obtained as filtrate, and the PRL was recovered from the retentate by washing the reversed filter unit with $100 \mu$ of 10 $\mathrm{mM}$ Tris, $\mathrm{pH} 7.4$ as per the manufacturer's protocol. In an identical experimental setup, the absorbance was measured from the buffer both inside and outside of the membrane. This 
control is kept to check whether degradation of the materials of the dialysis membrane is interfering with the assay. Each time, the released solution was returned after the spectra recording. Three independent experiments were performed for each sample.

\section{Cell proliferation assay using $\mathrm{Nb2}$ cells}

$\mathrm{Nb} 2$ cell line was grown in plastic culture flasks in RPMI medium supplemented with $10 \%$ heat-inactivated fetal bovine serum (FBS), $10 \%$ horse serum (HS), and $1 \mathrm{X}$ antibiotic solution and incubated at $37^{\circ} \mathrm{C}$ in a humidified incubator containing $5 \% \mathrm{CO}_{2}$ in the air. For proliferation assay, cells $\left(\sim 10^{5} /\right.$ well $)$ in a 96 well plate were seeded in RPMI medium in the presence of $1 \%$ fetal bovine serum and $10 \% \mathrm{HS}$ and incubated for $24 \mathrm{~h}$ to synchronize the cells at G0/G1 phase. After 24h, cells were treated with PRL monomer, monomer released from PRL-CSA and PRL-GAL at a dose range from $0.5-4 \mu \mathrm{M}$. The unrelated protein ovalbumin was used as the negative control. After incubation, cell proliferation was measured by MTT assay. To do so, $10 \mu \mathrm{l}$ of MTT solution ( $5 \mathrm{mg} / \mathrm{ml}$ in PBS) was added to the cells and incubated for $4 \mathrm{~h}$. Subsequently, $100 \mu \mathrm{l}$ of SDS-DMF solution (50\% DMF and 20\% SDS, pH 4.75) was added for overnight incubation. The absorption value of the product was measured at $560 \mathrm{~nm}$ and $690 \mathrm{~nm}$ as a background absorbance using a Spectramax M2e microplate reader (Molecular Devices, USA). The fold increases in cell proliferation compared to untreated cells were plotted against the concentration of the sample administered.

\section{Immunofluorescence}

Adult, female, Sprague-Dawley rats (200-250 g) taken for this study were maintained under the standard environmental conditions (12h: $12 \mathrm{~h}$, light: darkness cycle, chow and water ad libitum) and Institutional Animal Ethical Committee (IAEC) at NISER, Bhubaneswar, India approved the experimental protocols. First, the animals were anesthetized with sodium pentobarbital and then perfused transcardially with $50 \mathrm{ml}$ of $10 \mathrm{mM}$ phosphate buffer saline (PBS) $\mathrm{pH} 7.4$, followed by $50 \mathrm{ml}$ 4\% paraformaldehyde (PFA) in $100 \mathrm{mM}$ phosphate buffer $\mathrm{pH}$ 7.4. The pituitary glands were dissected out and post-fixed in $4 \%$ PFA overnight at $4^{\circ} \mathrm{C}$ 
followed by immersion in paraffin. Thin sections of the various paraffin-embedded tissues were made using a microtome. The sections were deparaffinized and rehydrated in decreasing concentrations of xylene and increasing concentrations of ethanol. The sections were rinsed twice in distilled water, followed by enzymatic antigen retrieval using $0.05 \%$ Trypsin incubation at $37^{\circ} \mathrm{C}$ for $5 \mathrm{~min}$. The sections were then washed with TBST buffer, $\mathrm{pH} 7.4$ (Trisbuffered saline containing $0.1 \%$ tween-20) followed by treatment with $0.2 \%$ Triton $\mathrm{X}-100$ for $10 \mathrm{~min}$. The sections were blocked using TBST containing 2\% BSA to prevent non-specific binding. PRL-GAL co-immuno-staining was performed using anti-PRL(Guinea pig polyclonal, from A. F. Parlow, National Hormone, and Pituitary Program, Harbor-ULCA Medical Center, Torrance, CA,1:1500) and anti-GAL primary antibody (Mouse, Abcam) (1:1500) overnight at $4^{\circ} \mathrm{C}$. Further, co-staining of pituitary tissue amyloids and GAL or PRL was performed using amyloid-specific (OC) antibody (rabbit polyclonal, Abcam, 1:500) and with respective hormone antibodies. All of the primary antibody-stained tissue slices were incubated overnight at $4{ }^{\circ} \mathrm{C}$ in a humidified chamber. The sections were rinsed in TBST and further incubated with the secondary antibody of goat anti-mouse FITC (1:500) or goat antirabbit Alexa Fluor-647(1:500) (Life Technologies, Thermo Scientific, USA) or goat antiGuinea pig Alexa Fluor 555(1:500)for $2 \mathrm{~h}$ at room temperature in a humid chamber. The sections were washed with TBST and mounted with a mounting medium. The sections were analyzed using a confocal microscope (Olympus IX81 combined with FV500) (Shinjuku, Tokyo, Japan) and images were recorded using a multi-channel image acquisition tool of Fluovision software (Zeiss, Oberkochen, Germany). For Thioflavin S (ThioS) staining, GAL and PRL staining was done using goat anti-mouse Alexa Fluor-555-conjugated secondary antibody (1:500 dilution) or goat anti-Guinea pig Alexa Fluor 555 respectively. The sections were then stained with $0.6 \%$ ThioS (Sigma-Aldrich) for $5 \mathrm{~min}$ in dark. The sections were washed with $50 \%$ ethanol for $2 \mathrm{~min}$ followed by TBST washing for $3 \mathrm{~min}$. The slides were then mounted in $90 \%$ glycerol and $10 \%$ phosphate-buffered saline (PBS) containing 1\% DABCO (1, 4-diazabicyclo-[2.2.2] octane, Sigma-Aldrich). The images and the sections were analyzed by multi-channel image acquisition tool of Fluovision software (Zeiss, Oberkochen, 
Germany) and Olympus FV-500 IX 81 confocal microscope (Shinjuku, Tokyo, Japan) respectively.

\section{Acknowledgments}

Authors wish to acknowledge Prof. P. S. Dannies and Prof. M. E. Hodsdon, Yale School of Medicine, USA for the plasmid of prolactin. Srivastav Ranganathan and Prem Prakash for the PRL schematic drawing and Congo red birefringence study, respectively. We are also thankful to CRNTS and IRCC, IIT Bombay for FTIR, electron microscopy, protein crystallography facilities and SPR facility. Authors wish to acknowledge DBT (BT/PR9797/NNT/28/774/2014) Government of India, Wadhwani research centre for Bioengineering (WRCB) and DBT/Welcome Trust India Alliance Fellowship [RD/0119DBTFL49-001] awarded to Shinjinee Sengupta for financial support.

\section{Author contributions}

D.C., R.S.J and S.K.M designed the experiments. D.C., R.S.J., S.R., A.N., N.S., S.S., L.G., P.K., D.D., A.P., C.P., S.K., P.S., and S.S performed experiments. All authors analyzed the data. S.K.M, D.C., S.R. \& R.S.J wrote the manuscript. All authors approved the final version of the manuscript.

\section{Conflict of interest}

The authors declare that they have no conflicts of interest with the contents of this article.

\section{Data availability statement}

The authors declare that all the data supporting the findings of this study are available within the paper and in supplementary information files. All the data analysis was performed using published tools and packages and has been cited in the paper and supplementary information text. 


\section{References}

1 Wszolek, Z. K., Uitti, R. J. \& Markopoulou, K. Familial Parkinson's disease and related conditions - Clinical genetics. Parkinson'S Disease 86, 33-43 (2001).

2 Katzman, R. \& Saitoh, T. Advances in Alzheimer's disease. FASEB J. 5, 278-286 (1991).

3 Talbot, N. J. Aerial morphogenesis: enter the chaplins. Curr Biol 13, R696-698, doi:S0960982203006432 [pii] (2003).

4 Destoumieux-Garzon, D. et al. Microcin E492 antibacterial activity: evidence for a TonB-dependent inner membrane permeabilization on Escherichia coli. Mol Microbiol 49, 1031-1041, doi:3610 [pii] (2003).

5 Oh, J. et al. Amyloidogenesis of type III-dependent harpins from plant pathogenic bacteria. J Biol Chem 282, 13601-13609, doi:M602576200 [pii] 10.1074/jbc.M602576200 [doi] (2007).

6 Maddelein, M. L., Dos Reis, S., Duvezin-Caubet, S., Coulary-Salin, B. \& Saupe, S. J. Amyloid aggregates of the HET-s prion protein are infectious. Proc. Natl. Acad. Sci. USA 99, 7402-7407 (2002).

7 Ritter, C. et al. Correlation of structural elements and infectivity of the HET-s prion. Nature 435, 844 - 848 (2005).

8 Berson, J. F., Harper, D. C., Tenza, D., Raposo, G. \& Marks, M. S. Pmel17 initiates premelanosome morphogenesis within multivesicular bodies. Mol Biol Cell 12, 34513464 (2001).

9 Watt, B. et al. N-terminal domains elicit formation of functional Pmel17 amyloid fibrils. $J$ Biol Chem 284, 35543-35555, doi:M109.047449 [pii] 10.1074/jbc.M109.047449 [doi] (2009).

10 Harper, D. C. et al. Premelanosome amyloid-like fibrils are composed of only golgiprocessed forms of Pmel17 that have been proteolytically processed in endosomes. $J$ Biol Chem 283, 2307-2322 (2008).

11 Barnhart, M. M. \& Chapman, M. R. Curli biogenesis and function. Annu. Rev. Microbiol. 60, 131-147 (2006).

12 Chapman, M. R. et al. Role of Escherichia coli curli operons in directing amyloid fiber formation. Science 295, 851-855, doi:10.1126/science.1067484 [doi] 295/5556/851 [pii] (2002).

13 Maji, S. K. et al. Functional amyloids as natural storage of peptide hormones in pituitary secretory granules. Science 325, 328-332, doi:1173155 [pii] 10.1126/science.1173155 [doi] (2009).

14 Jacob, R. S. et al. Amyloid formation of growth hormone in presence of zinc: Relevance to its storage in secretory granules. Sci Rep 6, 23370, doi:10.1038/srep23370 (2016).

15 Jacob, R., Anoop, A., Singh, P. \& Maji, S. in Protein Aggregation (ed Douglas A. Stein) in press. (Nova Science Publishers, Inc, 2010).

16 Maji, S. K. \& Riek, R. in Functional amyloid aggregation (ed S and Bucciantini Rigacci, M) 135-155 (Research Signpost, 2010).

17 Anoop, A. et al. Elucidating the role of disulfide bond on amyloid formation and fibril reversibility of somatostatin-14: Relevance to its storage and secretion. Journal of Biological Chemistry (2014).

18 Wood, S. J. et al. alpha-synuclein fibrillogenesis is nucleation-dependent Implications for the pathogenesis of Parkinson's disease. Journal of Biological Chemistry 274, 19509-19512 (1999). 
19 Srivastava, A. K. et al. beta-Amyloid aggregation and heterogeneous nucleation. Protein Sci 28, 1567-1581, doi:10.1002/pro.3674 (2019).

20 Eden, K., Morris, R., Gillam, J., MacPhee, C. E. \& Allen, R. J. Competition between primary nucleation and autocatalysis in amyloid fibril self-assembly. Biophys $J \mathbf{1 0 8}$, 632-643, doi:10.1016/j.bpj.2014.11.3465 (2015).

21 Arosio, P., Knowles, T. P. \& Linse, S. On the lag phase in amyloid fibril formation. Physical chemistry chemical physics : PCCP 17, 7606-7618, doi:10.1039/c4cp05563b (2015).

22 Ghosh, D. et al. The Parkinson, Äôs Disease-Associated H50Q Mutation Accelerates E \pm -Synuclein Aggregation in Vitro. Biochemistry 52, 6925-6927, doi:10.1021/bi400999d (2013).

23 Mehra, S. et al. Glycosaminoglycans have variable effects on alpha-synuclein aggregation and differentially affect the activities of the resulting amyloid fibrils. $J$ Biol Chem 293, 12975-12991, doi:10.1074/jbc.RA118.004267 (2018).

24 Alberti, S., Halfmann R Fau - Lindquist, S. \& Lindquist, S. Biochemical, cell biological, and genetic assays to analyze amyloid and prion aggregation in yeast.

25 Daskalov, A. et al. Structural and molecular basis of cross-seeding barriers in amyloids. Proc Natl Acad Sci U S A 118, doi:10.1073/pnas.2014085118 (2021).

26 Tornquist, M. et al. Secondary nucleation in amyloid formation. Chemical communications 54, 8667-8684, doi:10.1039/c8cc02204f (2018).

27 Buell, A. K. The growth of amyloid fibrils: rates and mechanisms. Biochem J 476, 2677-2703, doi:10.1042/BCJ20160868 (2019).

28 Koppen, J. et al. Amyloid-Beta Peptides Trigger Aggregation of Alpha-Synuclein In Vitro. Molecules 25, doi:10.3390/molecules25030580 (2020).

29 Nisbet, R. M., Polanco, J. C., Ittner, L. M. \& Gotz, J. Tau aggregation and its interplay with amyloid-beta. Acta neuropathologica 129, 207-220, doi:10.1007/s00401-014-1371-2 (2015).

30 Bennett, R. E. et al. Enhanced Tau Aggregation in the Presence of Amyloid beta. Am J Pathol 187, 1601-1612, doi:10.1016/j.ajpath.2017.03.011 (2017).

31 St-Amour, I., Turgeon, A., Goupil, C., Planel, E. \& Hebert, S. S. Co-occurrence of mixed proteinopathies in late-stage Huntington's disease. Acta neuropathologica 135, 249-265, doi:10.1007/s00401-017-1786-7 (2018).

32 Spires-Jones, T. L., Attems, J. \& Thal, D. R. Interactions of pathological proteins in neurodegenerative diseases. Acta neuropathologica 134, 187-205, doi:10.1007/s00401-017-1709-7 (2017).

33 Bharadwaj, P. et al. The Link between Type 2 Diabetes and Neurodegeneration: Roles for Amyloid-beta, Amylin, and Tau Proteins. Journal of Alzheimer's disease : JAD 59, 421-432, doi:10.3233/JAD-161192 (2017).

34 Biessels, G. J., Kappelle, L. J. \& Utrecht Diabetic Encephalopathy Study, G. Increased risk of Alzheimer's disease in Type II diabetes: insulin resistance of the brain or insulin-induced amyloid pathology? Biochemical Society transactions 33, 1041-1044, doi:10.1042/BST0331041 (2005).

35 Koshiyama, H. et al. Central galanin stimulates pituitary prolactin secretion in rats: possible involvement of hypothalamic vasoactive intestinal polypeptide. Neuroscience letters 75, 49-54, doi:10.1016/0304-3940(87)90073-5 (1987).

36 Murakami, Y., Ohshima, K., Mochizuki, T. \& Yanaihara, N. Effect of human galanin on growth hormone prolactin, and antidiuretic hormone secretion in normal men. The Journal of clinical endocrinology and metabolism 77, 1436-1438, doi:10.1210/jcem.77.5.7521348 (1993). 
37 Wynick, D. et al. Galanin regulates prolactin release and lactotroph proliferation. Proc Natl Acad Sci U S A 95, 12671-12676, doi:10.1073/pnas.95.21.12671 (1998).

38 Hyde, J. F., Engle, M. G. \& Maley, B. E. Colocalization of galanin and prolactin within secretory granules of anterior pituitary cells in estrogen-treated Fischer 344 rats. Endocrinology 129, 270-276 (1991).

39 Koshiyama, H. et al. Galanin interacts with serotonin in stimulating prolactin secretion in the rat. Journal of neuroendocrinology 2, 217-220, doi:10.1111/j.13652826.1990.tb00853.x (1990).

40 Koshiyama, H. et al. Galanin-induced prolactin release in rats: pharmacological evidence for the involvement of alpha-adrenergic and opioidergic mechanisms. Brain research 507, 321-324, doi:10.1016/0006-8993(90)90290-r (1990).

41 Teilum, K. et al. Solution structure of human prolactin. J Mol Biol 351, 810-823, doi:10.1016/j.jmb.2005.06.042 (2005).

42 Keeler, C., Dannies, P. S. \& Hodsdon, M. E. The tertiary structure and backbone dynamics of human prolactin. J Mol Biol 328, 1105-1121 (2003).

43 Evans, H. F. \& Shine, J. Human galanin: molecular cloning reveals a unique structure. Endocrinology 129, 1682-1684, doi:10.1210/endo-129-3-1682 (1991).

44 Bersani, M. et al. Human galanin: primary structure and identification of two molecular forms. FEBS Lett 283, 189-194, doi:10.1016/0014-5793(91)80585-q (1991).

45 Fernandez-Escamilla, A. M., Rousseau, F., Schymkowitz, J. \& Serrano, L. Prediction of sequence-dependent and mutational effects on the aggregation of peptides and proteins. Nat Biotechnol 22, 1302-1306, doi:10.1038/nbt1012 (2004).

46 Bussiere, T. et al. Morphological characterization of Thioflavin-S-positive amyloid plaques in transgenic Alzheimer mice and effect of passive Abeta immunotherapy on their clearance. Am J Pathol 165, 987-995, doi:10.1016/s0002-9440(10)63360-3 (2004).

47 Sunde, M. et al. Common core structure of amyloid fibrils by synchrotron X-ray diffraction. J Mol Biol 273, 729-739 (1997).

48 Sunde, M. \& Blake, C. The structure of amyloid fibrils by electron microscopy and Xray diffraction. Adv Protein Chem 50, 123-159 (1997).

49 Jackson, M. \& Mantsch, H. H. The use and misuse of FTIR spectroscopy in the determination of protein structure. Critical Reviews in Biochemistry and Molecular Biology 30, 95-120 (1995).

50 Kong, J. \& Yu, S. Fourier transform infrared spectroscopic analysis of protein secondary structures. Acta biochimica et biophysica Sinica 39, 549-559, doi:10.1111/j.1745-7270.2007.00320.x (2007).

51 Ren, B. et al. Fundamentals of cross-seeding of amyloid proteins: an introduction. Journal of materials chemistry. B 7, 7267-7282, doi:10.1039/c9tb01871a (2019).

52 Ivanova, M. I., Lin, Y., Lee, Y. H., Zheng, J. \& Ramamoorthy, A. Biophysical processes underlying cross-seeding in amyloid aggregation and implications in amyloid pathology. Biophysical chemistry 269, 106507, doi:10.1016/j.bpc.2020.106507 (2021).

53 Hartman, K. et al. Bacterial curli protein promotes the conversion of PAP248-286 into the amyloid SEVI: cross-seeding of dissimilar amyloid sequences. PeerJ 1, e5, doi:10.7717/peerj.5 (2013).

54 Morales, R., Moreno-Gonzalez, I. \& Soto, C. Cross-seeding of misfolded proteins: implications for etiology and pathogenesis of protein misfolding diseases. PLoS pathogens 9, e1003537, doi:10.1371/journal.ppat.1003537 (2013). 
bioRxiv preprint doi: https://doi.org/10.1101/2021.08.31.458467; this version posted September 1,2021 . The copyright holder for this preprint (which was not certified by peer review) is the author/funder, who has granted bioRxiv a license to display the preprint in perpetuity. It is made available under aCC-BY-NC-ND 4.0 International license.

55 Linse, S. Monomer-dependent secondary nucleation in amyloid formation. Biophysical reviews 9, 329-338, doi:10.1007/s12551-017-0289-z (2017).

56 Koloteva-Levine, N. et al. Amyloid particles facilitate surface-catalyzed crossseeding by acting as promiscuous nanoparticles. bioRxiv, 2020.2009.2001.278481, doi:10.1101/2020.09.01.278481 (2020).

57 Sarell, C. J., Stockley, P. G. \& Radford, S. E. Assessing the causes and consequences of co-polymerization in amyloid formation. Prion 7, 359-368, doi:10.4161/pri.26415 (2013).

58 Cohen, S. I. A. et al. Distinct thermodynamic signatures of oligomer generation in the aggregation of the amyloid-beta peptide. Nature chemistry 10, 523-531, doi:10.1038/s41557-018-0023-x (2018).

59 Ranganathan, S. et al. Molecular interpretation of ACTH-beta-endorphin coaggregation: relevance to secretory granule biogenesis. PLoS One 7, e31924, doi:10.1371/journal.pone.0031924 (2012).

60 Schmidt, W. E. et al. Isolation and primary structure of pituitary human galanin, a 30residue nonamidated neuropeptide. Proc Natl Acad Sci U S A 88, 11435-11439, doi:10.1073/pnas.88.24.11435 (1991).

61 Nilsson, C. L., Brinkmalm, A., Minthon, L., Blennow, K. \& Ekman, R. Processing of neuropeptide Y, galanin, and somatostatin in the cerebrospinal fluid of patients with Alzheimer's disease and frontotemporal dementia. Peptides 22, 2105-2112 (2001).

62 Cino, E. A., Choy, W. Y. \& Karttunen, M. Comparison of Secondary Structure Formation Using 10 Different Force Fields in Microsecond Molecular Dynamics Simulations. Journal of chemical theory and computation 8, 2725-2740, doi:10.1021/ct300323g (2012).

63 Oostenbrink, C., Villa, A., Mark, A. E. \& van Gunsteren, W. F. A biomolecular force field based on the free enthalpy of hydration and solvation: the GROMOS force-field parameter sets 53A5 and 53A6. Journal of computational chemistry 25, 1656-1676, doi:10.1002/jcc.20090 (2004).

64 Tian, C. et al. ff19SB: Amino-Acid-Specific Protein Backbone Parameters Trained against Quantum Mechanics Energy Surfaces in Solution. Journal of chemical theory and computation 16, 528-552, doi:10.1021/acs.jctc.9b00591 (2020).

65 Bemporad, F. \& Chiti, F. Protein misfolded oligomers: experimental approaches, mechanism of formation, and structure-toxicity relationships. Chem Biol 19, 315-327, doi:10.1016/j.chembiol.2012.02.003 (2012).

66 Cascella, R. et al. The release of toxic oligomers from alpha-synuclein fibrils induces dysfunction in neuronal cells. Nat Commun 12, 1814, doi:10.1038/s41467-02121937-3 (2021).

67 Bulatov, A. A., Osipova, T. A. \& Raevskaia, G. V. [Proliferative effect of prolactin and growth hormone from human serum in cultured rat lymphoma $\mathrm{Nb} 2$ cells]. Biulleten' eksperimental'noi biologii i meditsiny 122, 559-563 (1996).

68 Lebrun, J. J., Ali, S., Sofer, L., Ullrich, A. \& Kelly, P. A. Prolactin-induced proliferation of $\mathrm{Nb} 2$ cells involves tyrosine phosphorylation of the prolactin receptor and its associated tyrosine kinase JAK2. J Biol Chem 269, 14021-14026 (1994).

69 Upadhyay, V., Singh, A., Jha, D., Singh, A. \& Panda, A. K. Recovery of bioactive protein from bacterial inclusion bodies using trifluoroethanol as solubilization agent. Microbial cell factories 15, 100, doi:10.1186/s12934-016-0504-9 (2016).

70 Maji, S. K., Wang, L., Greenwald, J. \& Riek, R. Structure-activity relationship of amyloid fibrils. FEBS Lett 583, 2610-2617, doi:S0014-5793(09)00528-6 [pii] 10.1016/j.febslet.2009.07.003 (2009). 
71 Chiti, F. \& Dobson, C. M. Protein misfolding, functional amyloid, and human disease. Annu Rev Biochem 75, 333-366 (2006).

72 Fowler, D. M., Koulov, A. V., Balch, W. E. \& Kelly, J. W. Functional amyloid--from bacteria to humans. Trends Biochem Sci 32, 217-224 (2007).

73 Waxman, E. A. \& Giasson, B. I. Induction of intracellular tau aggregation is promoted by alpha-synuclein seeds and provides novel insights into the hyperphosphorylation of tau. The Journal of neuroscience : the official journal of the Society for Neuroscience 31, 7604-7618, doi:10.1523/JNEUROSCI.0297-11.2011 (2011).

74 Moussaud, S. et al. Alpha-synuclein and tau: teammates in neurodegeneration? Mol Neurodegener 9, 43, doi:10.1186/1750-1326-9-43 (2014).

75 Bassil, F. et al. Amyloid-Beta (Abeta) Plaques Promote Seeding and Spreading of Alpha-Synuclein and Tau in a Mouse Model of Lewy Body Disorders with Abeta Pathology. Neuron 105, 260-275 e266, doi:10.1016/j.neuron.2019.10.010 (2020).

76 Reggio, H. A. \& Palade, G. E. Sulfated compounds in the zymogen granules of the guinea pig pancreas. J Cell Biol 77, 288-314 (1978).

77 Zanini, A. et al. Molecular organization of prolactin granules. II. Characterization of glycosaminoglycans and glycoproteins of the bovine prolactin matrix. J Cell Biol 86, 260-272 (1980).

78 Krebs, M. R., Morozova-Roche, L. A., Daniel, K., Robinson, C. V. \& Dobson, C. M. Observation of sequence specificity in the seeding of protein amyloid fibrils. Protein Sci 13, 1933-1938, doi:10.1110/ps.04707004 (2004).

79 Walker, L. C., Diamond, M. I., Duff, K. E. \& Hyman, B. T. Mechanisms of protein seeding in neurodegenerative diseases. JAMA neurology 70, 304-310, doi:10.1001/jamaneurol.2013.1453 (2013).

80 O'Nuallain, B., Williams, A. D., Westermark, P. \& Wetzel, R. Seeding specificity in amyloid growth induced by heterologous fibrils. J Biol Chem 279, 17490-17499, doi:10.1074/jbc.M311300200 (2004).

$81 \mathrm{Yu}, \mathrm{X}$. et al. Cross-seeding and conformational selection between three- and fourrepeat human Tau proteins. J Biol Chem 287, 14950-14959, doi:10.1074/jbc.M112.340794 (2012).

82 Sambashivan, S., Liu, Y., Sawaya, M. R., Gingery, M. \& Eisenberg, D. Amyloid-like fibrils of ribonuclease A with three-dimensional domain-swapped and native-like structure. Nature 437, 266-269, doi:10.1038/nature03916 (2005).

83 Sankoorikal, B. J., Zhu, Y. L., Hodsdon, M. E., Lolis, E. \& Dannies, P. S. Aggregation of human wild-type and H27A-prolactin in cells and in solution: roles of $\mathrm{Zn}(2+), \mathrm{Cu}(2+)$, and pH. Endocrinology 143, 1302-1309 (2002).

84 Maji, S. K. et al. Amino acid position-specific contributions to amyloid beta-protein oligomerization. $J$ Biol Chem 284, 23580-23591, doi:M109.038133 [pii] 10.1074/jbc.M109.038133 (2009).

85 van Zundert, G. C. P. et al. The HADDOCK2.2 Web Server: User-Friendly Integrative Modeling of Biomolecular Complexes. J Mol Biol 428, 720-725, doi:10.1016/j.jmb.2015.09.014 (2016).

86 Maji, S. K. et al. Amyloid as a depot for the formulation of long-acting drugs. Plos Biology 6, e17 (2008). 